\title{
Front Matter: Volume 9148
}

"Front Matter: Volume 9148," Proc. SPIE 9148, Adaptive Optics Systems IV, 914801 (5 September 2014); doi: 10.1117/12.2076627

SPIE Event: SPIE Astronomical Telescopes + Instrumentation, 2014, Montréal, Quebec, Canada 


\title{
PROCEEDINGS OF SPIE
}

\section{Adaptive Optics Systems IV}

\author{
Enrico Marchetti \\ Laird M. Close \\ Jean-Pierre Véran \\ Editors
}

22-27 June 2014

Montréal, Canada

Sponsored by

SPIE

Cooperating Organizations

American Astronomical Society (United States) - Australian Astronomical Observatory (Australia) - Association of Universities for Research in Astronomy (AURA) • Canadian Astronomical Society (CASCA) (Canada) • Canadian Space Agency (Canada) • European Astronomical Society (Switzerland) • European Southern Observatory (Germany) • National Radio Astronomy Observatory • Royal Astronomical Society (United Kingdom) • Science \& Technology Facilities Council (United Kingdom)

Published by

SPIE

Volume 9148

Part One of Three Parts

Proceedings of SPIE 0277-786X, V. 9148

SPIE is an international society advancing an interdisciplinary approach to the science and application of light.

Adaptive Optics Systems IV, edited by Enrico Marchetti, Laird M. Close,

Jean-Pierre Véran, Proc. of SPIE Vol. 9148, 914801 - (c) 2014 SPIE

CCC code: $0277-786 \mathrm{X} / 14 / \$ 18 \cdot$ doi: $10.1117 / 12.2076627$

Proc. of SPIE Vol. $9148914801-1$ 
The papers included in this volume were part of the technical conference cited on the cover and title page. Papers were selected and subject to review by the editors and conference program committee. Some conference presentations may not be available for publication. The papers published in these proceedings reflect the work and thoughts of the authors and are published herein as submitted. The publisher is not responsible for the validity of the information or for any outcomes resulting from reliance thereon.

Please use the following format to cite material from this book:

Author(s), "Title of Paper," in Adaptive Optics Systems IV, edited by Enrico Marchetti, Laird M. Close, Jean-Pierre Véran, Proceedings of SPIE Vol. 9148 (SPIE, Bellingham, WA, 2014) Article CID Number.

ISSN: 0277-786X

ISBN: 9780819496164

Published by

SPIE

P.O. Box 10, Bellingham, Washington 98227-0010 USA

Telephone +1 3606763290 (Pacific Time) · Fax +1 3606471445

SPIE.org

Copyright (C) 2014, Society of Photo-Optical Instrumentation Engineers.

Copying of material in this book for internal or personal use, or for the internal or personal use of specific clients, beyond the fair use provisions granted by the U.S. Copyright Law is authorized by SPIE subject to payment of copying fees. The Transactional Reporting Service base fee for this volume is $\$ 18.00$ per article (or portion thereof), which should be paid directly to the Copyright Clearance Center (CCC), 222 Rosewood Drive, Danvers, MA 01923. Payment may also be made electronically through CCC Online at copyright.com. Other copying for republication, resale, advertising or promotion, or any form of systematic or multiple reproduction of any material in this book is prohibited except with permission in writing from the publisher. The CCC fee code is 0277-786X/14/\$18.00.

Printed in the United States of America.

Publication of record for individual papers is online in the SPIE Digital Library.

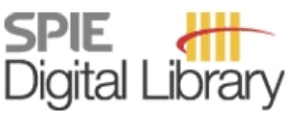

SPIEDigitalLibrary.org

Paper Numbering: Proceedings of SPIE follow an e-First publication model, with papers published first online and then in print and on CD-ROM. Papers are published as they are submitted and meet publication criteria. A unique, consistent, permanent citation identifier (CID) number is assigned to each article at the time of the first publication. Utilization of CIDs allows articles to be fully citable as soon as they are published online, and connects the same identifier to all online, print, and electronic versions of the publication. SPIE uses a six-digit CID article numbering system in which:

- The first four digits correspond to the SPIE volume number.

- The last two digits indicate publication order within the volume using a Base 36 numbering

system employing both numerals and letters. These two-number sets start with 00, 01, 02, 03, 04, $05,06,07,08,09,0 A, 0 B \ldots$. 0Z, followed by 10-1Z, 20-2Z, etc.

The CID Number appears on each page of the manuscript. The complete citation is used on the first page, and an abbreviated version on subsequent pages. Numbers in the index correspond to the last two digits of the six-digit CID Number. 


\section{Contents}

xxxv Conference Committee

\section{Part One}

914802 ESO adaptive optics facility progress and first laboratory test results (Invited Paper) [9148-1] R. Arsenault, P.-Y. Madec, J. Paufique, P. La Penna, S. Stroebele, E. Vernet, J.-F. Pirard, W. Hackenberg, H. Kuntschner, J. Kolb, N. Muller, A. Garcia-Rissmann, M. Le Louarn, P. Amico, N. Hubin, J.-L. Lizon, R. Ridings, P. Haguenaver, J. A. Abad, G. Fischer, V. Heinz, M. Kiekebusch, J. Argomedo, R. Conzelmann, S. Tordo, R. Donaldson, C. Soenke, P. Duhoux, E. Fedrigo, B. Delabre, A. Jost, M. Duchateau, M. Downing, J. Reyes Moreno, A. Manescau, D. Bonaccini Calia, M. Quattri, C. Dupuy, I. M. Guidolin, M. Comin, R. Guzman, B. Buzzoni, J. Quentin, S. Lewis, P. Jolley, M. Kraus, T. Pfrommer, European Southern Observatory (Germany); R. Biasi, Microgate S.r.l. (Italy); D. Gallieni, A.D.S. International S.r.l. (Italy); R. Stuik, Leiden Observatory (Netherlands); W. Kaenders, B. Ernstberger, A. Friedenaver, TOPTICA Photonics AG (Germany)

914803 Large binocular telescope interferometer adaptive optics: on-sky performance and lessons learned [9148-2]

V. P. Bailey, P. M. Hinz, Steward Observatory, The Univ. of Arizona (United States);

A. T. Puglisi, S. Esposito, INAF - Osservatorio Astrofisico di Arcetri (Italy); V. Vaitheeswaran, A. J. Skemer, D. Defrère, A. Vaz, J. M. Leisenring, Steward Observatory, The Univ. of Arizona (United States)

914804 MagAO: Status and on-sky performance of the Magellan adaptive optics system [9148-3] K. M. Morzinski, L. M. Close, J. R. Males, Steward Observatory, The Univ. of Arizona (United States); D. Kopon, Max-Planck-Institut für Astronomie (Germany); P. M. Hinz, Steward Observatory, The Univ. of Arizona (United States); S. Esposito, A. Riccardi, A. Puglisi, E. Pinna, R. Briguglio, M. Xompero, F. Quirós-Pacheco, INAF - Osservatorio Astrofisico di Arcetri (Italy); V. Bailey, K. B. Follette, Steward Observatory, The Univ. of Arizona (United States); T. J. Rodigas, Carnegie Institution of Washington (United States); Y.-L. Wu, Steward Observatory, The Univ. of Arizona (United States); C. Arcidiacono, INAF - Osservatorio Astronomico di Bologna (Italy); J. Argomedo, European Southern Observatory (Germany); L. Busoni, INAF - Osservatorio Astrofisico di Arcetri (Italy); T. Hare, A. Uomoto, Carnegie Observatories (United States); A. Weinberger, Carnegie Institution of Washington (United States)

914805 ShaneAO: wide science spectrum adaptive optics system for the Lick Observatory [9148-76]

D. Gavel, R. Kupke, D. Dillon, A. Norton, C. Ratliff, J. Cabak, A. Phillips, C. Rockosi, R. McGurk, S. Srinath, M. Peck, W. Deich, K. Lanclos, J. Gates, M. Saylor, J. Ward, T. Pfister, Univ. of California Observatories (United States) 
914807 Series production of next-generation guide-star lasers at TOPTICA and MPBC [9148-6] M. Enderlein, A. Friedenaver, R. Schwerdt, P. Rehme, TOPTICA Photonics AG (Germany); D. Wei, V. Karpov, MPB Communications Inc. (Canada); B. Ernstberger, P. Leisching, TOPTICA Photonics AG (Germany); W. R. L. Clements, MPB Communications Inc. (Canada); W. G. Kaenders, TOPTICA Photonics AG (Germany)

$914808 \quad$ Laser guide star facility developments at W. M. Keck Observatory [9148-7]

J. C. Y. Chin, P. Wizinowich, E. Wetherell, S. Cetre, S. Ragland, R. Campbell, S. Lilley, J. Lyke, D. Medeiros, R. Rampy, T. Stalcup, K. Tsubota, P. Tucker, K. Wei, W. M. Keck Observatory (United States)

\section{SESSION $3 \quad$ ASTRONOMY WITH AO I}

$91480 \mathrm{~A}$ Twelve thousand laser-AO observations: first results from the Robo-AO large surveys [9148-9]

N. M. Law, The Univ. of North Carolina at Chapel Hill (United States); C. Baranec, Institute for Astronomy, Univ. of Hawai'i (United States); R. L. Riddle, California Institute of Technology (United States)

9148 OB Near-infrared astrometry of star clusters with different flavors of adaptive optics and HST (Invited Paper) [9148-10]

J. R. Lu, Institute for Astronomy, Univ. of Hawai'i (United States); B. Neichel, Lab. d'Astrophysique de Marseille, CNRS, Aix Marseille Univ. (France); J. Anderson, Space Telescope Science Institute (United States); E. Sinukoff, M. W. Hosek Jr., Institute for Astronomy, Univ. of Hawai'i (United States); A. M. Ghez, Univ. of California, Los Angeles (United States); F. Rigaut, The Australian National Univ. (Australia)

9148 OC Galactic astronomy with AO: nearby star clusters and moving groups (Invited Paper) [9148-11]

T. J. Davidge, Dominion Astrophysical Observatory (Canada)

9148 OD Supernovae and extragalactic astronomy with laser guide star adaptive optics (Invited Paper) [9148-12]

S. D. Ryder, Australian Astronomical Observatory (Australia); S. Mattila, E. Kankare, Univ. of Turku (Finland); P. Vaisanen, South African Astronomical Observatory (South Africa)

\section{SESSION 4 WAVEFRONT CORRECTION I}

9148 OF Living with adaptive secondary mirrors 365/7/24 [9148-14]

J. C. Christou, G. Brusa, J. C. Guerra, M. Lefebvre, D. Miller, G. Rahmer, R. Sosa, M. Wagner, Large Binocular Telescope Observatory (United States)

9148 OG Recent results and future DMs for astronomy and for space applications at CILAS [9148-15] J.-C. Sinquin, A. Bastard, E. Beaufort, CILAS (France); T. Berkefeld, Kiepenhever-Institut für Sonnenphysik (Germany); L. Cadiergues, V. Costes, Ctr. National d'Etudes Spatiales (France); R. Cousty, CILAS (France); C. Dekhtiar, F. Di Gesu, Airbus Defence and Space 
(France); X. Gilbert, C. Grèzes-Besset, D. Groeninck, CILAS (France); M. Hartung, Gemini Observatory (Chile); H. Krol, A. Moreau, P. Morin, H. Pagès, R. Palomo, CILAS (France); G. Scharmer, Stockholm Univ. (Sweden); D. Soltau, Kiepenhever-Institut für Sonnenphysik (Germany); J.-P. Véran, National Research Council Canada (Canada)

$9148 \mathrm{OH}$ Piezoelectric deformable mirror technologies for astronomy at IOE, CAS [9148-16] C. Guan, X. Fan, X. Zhang, H. Zhou, J. Mu, L. Xue, K. Wei, H. Xian, C. Rao, Y. Zhang, N. Ling, Institute of Optics and Electronics (China) and The Key Lab. On Adaptive Optics (China)

9148 0J The Gemini planet imager: first light and commissioning (Invited Paper) [9148-18] B. A. Macintosh, Stanford Univ. (United States) and Lawrence Livermore National Lab. (United States); A. Anthony, J. Atwood, NRC - Herzberg Institute of Astrophysics (Canada); B. Bauman, Lawrence Livermore National Lab. (United States); A. Cardwell, Gemini Observatory (United States); K. Caputa, NRC - Herzberg Institute of Astrophysics (Canada); J. Chilcote, Univ. of California, Los Angeles (United States); R. J. De Rosa, Arizona State Univ. (United States); D. Dillon, Univ. of California, Berkeley (United States); R. Doyon, Univ. de Montréal (Canada); J. Dunn, NRC - Herzburg Institute of Astrophysics (Canada); D. Erickson, Lab. for Atmospheric and Space Physics (United States); M. P. Fitzgerald, Univ. of California, Los Angeles (United States); D. T. Gavel, Univ. of California, Santa Cruz (United States); R. Galvez, S. Goodsell, Gemini Observatory (United States); J. Graham, Univ. of California, Berkeley (United States); A. Z. Greenbaum, Johns Hopkins Univ. (United States); M. Hartung, P. Hibon, Gemini Observatory (United States); P. Ingraham, Stanford Univ. (United States); D. Kerley, NRC - Herzberg Institute of Astrophysics (Canada);

Q. Konopacky, Dunlap Institute, Univ. of Toronto (Canada); K. Labrie, Gemini Observatory (United States); J. Larkin, Univ. of California, Los Angeles (United States); J. Maire, Dunlap Institute, Univ. Of Toronto (Canada); F. Marchis, SETI Institute (United States); C. Marois, NRC - Herzburg Institute of Astrophysics (Canada); M. Millar-Blanchaer, Dunlap Institute, Univ. of Toronto (Canada); K. Morzinski, Stewart Observatory, The Univ. of Arizona (United States); A. Nunez, Gemini Observatory (United States); R. Oppenheimer, American Museum of Natural History (United States); D. Palmer, Lawrence Livermore National Lab. (United States); J. Pazder, NRC - Herzberg Institute of Astrophysics (Canada); M. Perrin, Space Telescope Science Institute (United States); L. A. Poyneer, Lawrence Livermore National Lab. (United States); L. Pueyo, Space Telescope Science Institute (United States); C. Quiroz, F. Rantakyro, Gemini Observatory (United States); V. Reshetov, L. Saddlemyer, NRC Herzberg Institute of Astrophysics (Canada); N. Sadakuni, Gemini Observatory (United States); D. Savransky, Cornell Univ. (United States); A. Serio, Gemini Observatory (United States); A. Sivaramakrishnan, Space Telescope Science Institute (United States); M. Smith, NRC - Herzberg Institute of Astrophysics (Canada); R. Soummer, Space Telescope Science Institute (United States); S. Thomas, NASA Ames Research Ctr. (United States); J. K. Wallace, Jet Propulsion Lab. (United States); J. Wang, Univ. of California, Berkeley (United States); J. Weiss, Univ. of California, Los Angeles (United States); S. Wiktorowicz, Univ. of California, Santa Cruz (United States); S. G. Wolff, Johns Hopkins Univ. (United States)

9148 OK On-sky performance during verification and commissioning of the Gemini Planet Imager's adaptive optics system [9148-19]

L. A. Poyneer, Lawrence Livermore National Lab. (United States); R. J. De Rosa, Arizona State Univ. (United States) and Univ. of Exeter (United Kingdom); B. Macintosh, Kavli Institute for Particle Astrophysics and Cosmology, Stanford Univ. (United States) and Lawrence 
Livermore National Lab. (United States); D. W. Palmer, Lawrence Livermore National Lab. (United States); M. D. Perrin, Space Telescope Science Institute (United States);

N. Sadakuni, Gemini Observatory (Chile); D. Savransky, Cornell Univ. (United States); B. Bauman, Lawrence Livermore National Lab. (United States); A. Cardwell, Gemini Observatory (Chile); J. K. Chilcote, Univ. of California, Los Angeles (United States); D. Dillon, D. Gavel, Univ. of California, Santa Cruz (United States); S. J. Goodsell, M. Hartung, P. Hibon, F. T. Rantakyrö, Gemini Observatory (Chile); S. Thomas, NASA Ames Research Ctr. (United States) and Univ. of California, Santa Cruz (United States); J.-P. Véran, NRC Herzberg Institute of Astrophysics (Canada)

$9148 \mathrm{OL} \quad H i g h$ contrast imaging at the LBT: the LEECH exoplanet imaging survey [9148-20] A. J. Skemer, P. Hinz, Steward Observatory, The Univ. of Arizona (United States); S. Esposito, INAF - Osservatorio Astrofisico di Arcetri (Italy); M. F. Skrutskie, Univ. of Virginia (United States); D. Defrère, V. Bailey, J. Leisenring, D. Apai, Steward Observatory, The Univ. of Arizona (United States); B. Biller, Max-Planck-Institut für Astronomie (Germany) and Univ. of Edinburgh (United Kingdom); M. Bonnefoy, Max-Planck-Institut für Astronomie (Germany) and IPAG (France); W. Brandner, E. Buenzli, Max-Planck-Institut für Astronomie (Germany); L. Close, Steward Observatory, The Univ. of Arizona (United States); J. Crepp, Univ. of Notre Dame (United States); R. J. De Rosa, Arizona State Univ. (United States) and Univ. of Exeter (United Kingdom); S. Desidera, INAF - Osservatorio Astronomico di Padova (Italy); J. Eisner, Steward Observatory, The Univ. of Arizona (United States); J. Fortney, Univ. of California, Santa Cruz (United States); T. Henning, Max-Planck-Institut für Astronomie (Germany); K.-H. Hofmann, Max-Planck-Institut für Radioastronomie (Germany); T. Kopytova, MaxPlanck-Institut für Astronomie (Germany); A.-L. Maire, INAF - Osservatorio Astronomico di Padova (Italy); J. R. Males, Steward Observatory, The Univ. of Arizona (United States); R. Millan-Gabet, NASA Exoplanet Science Institute (United States); K. Morzinski, Steward Observatory, The Univ. of Arizona (United States); A. Oza, Univ. of Virginia (United States); J. Patience, A. Rajan, Arizona State Univ. (United States); G. Rieke, Steward Observatory, The Univ. of Arizona (United States); D. Schertl, Max-Planck-Institut für Radioastronomie (Germany); J. Schlieder, Max-Planck-Institut für Astronomie (Germany); K. Su, A. Vaz, Steward Observatory, The Univ. of Arizona (United States); K. Ward-Duong, Arizona State Univ. (United States); G. Weigelt, Max-Planck-Institut für Radioastronomie (Germany); C. E. Woodward, Univ. of Minnesota (United States); N. Zimmerman, Max-Planck-Institut für Astronomie (Germany) and Princeton Univ. (United States)

9148 OM The VORTEX project: first results and perspectives [9148-21]

O. Absil, Univ. de Liège (Belgium); D. Mawet, European Southern Observatory (Chile);

C. Delacroix, Univ. de Liège (Belgium); P. Forsberg, M. Karlsson, Uppsala Univ. (Sweden); S. Habraken, J. Surdej, Univ. de Liège (Belgium); P.-A. Absil, Univ. Catholique de Louvain (Belgium); B. Carlomagno, Univ. de Liège (Belgium); V. Christiaens, Univ. de Chile (Chile) and Univ. de Liège (Belgium); D. Defrère, Steward Observatory, The Univ. of Arizona (United States); C. A. Gomez Gonzalez, E. Huby, A. Jolivet, Univ. de Liège (Belgium); J. Milli, European Southern Observatory (Chile); P. Piron, Univ. de Liège (Belgium); E. Vargas Catalan, Uppsala Univ. (Sweden); M. Van Droogenbroeck, Univ. de Liège (Belgium)

\section{SESSION 6 ADVANCES IN AO CONTROL I}

9148 ON On-sky vibration environment for the Gemini Planet Imager and mitigation effort [9148-175] M. Hartung, T. Hayward, Gemini Observatory (Chile); L. Saddlemyer, NRC - Herzburg Institute of Astrophysics (Canada); L. Poyneer, Lawrence Livermore National Lab. (United States); A. Cardwell, Gemini Observatory (Chile); C. Cavedoni, Gemini Observatory 
(United States); M. Cho, National Optical Astronomy Observatory (United States);

J. K. Chilcote, Univ. of California, Los Angeles (United States); P. Collins, Gemini Observatory

(Chile); D. Dillon, Univ. of California, Santa Cruz (United States); R. Galvez, G. Gausachs,

Gemini Observatory (Chile); S. Goodsell, Gemini Observatory (United States);

A. Guesalaga, Pontificia Univ. Católica de Chile (Chile); P. Hibon, Gemini Observatory (Chile); J. Larkin, Univ. of California, Los Angeles (United States); B. Macintosh, Kavli Institute for Particle Astrophysics and Cosmology, Stanford Univ. (United States); D. Palmer, Lawrence Livermore National Lab. (United States); N. Sadakuni, Gemini Observatory (Chile); D. Savransky, Lawrence Livermore National Lab. (United States); A. Serio, F. Rantakyrö, Gemini Observatory (Chile); K. Wallace, Jet Propulsion Lab. (United States)

914800 SPHERE eXtreme AO control scheme: final performance assessment and on sky validation of the first auto-tuned LQG based operational system [9148-23]

C. Petit, J.-F. Sauvage, ONERA (France); T. Fusco, ONERA (France) and Lab.

d'Astrophysique de Marseille, CNRS (France); A. Sevin, Observatoire de Paris, CNRS (France); M. Suarez, European Southern Observatory (Germany); A. Costille, A. Vigan, Lab. d'Astrophysique de Marseille, CNRS (France); C. Soenke, European Southern Observatory (Germany); D. Perret, Observatoire de Paris, CNRS (France); S. Rochat, Institut de Planétologie et d'Astrophysique de Grenoble, CNRS (France); A. Barrufolo, B. Salasnich, INAF (Italy); J.-L. Beuzit, Institut de Planétologie et d'Astrophysique de Grenoble, CNRS (France); K. Dohlen, Lab. d'Astrophysique de Marseille, CNRS (France); D. Movillet, P. Puget, Institut de Planétologie et d'Astrophysique de Grenoble, CNRS (France); F. Wildi, Univ. of Genève (Switzerland); M. Kasper, European Southern Observatory (Germany); J.-M. Conan, ONERA (France); C. Kulcsár, H.-F. Raynaud, Lab. Charles Fabry, CNRS, Institut d'Optique Graduate School (France)

$9148 \mathrm{OP}$ Fast algorithm for wavefront reconstruction in XAO/SCAO with pyramid wavefront sensor [9148-24]

I. Shatokhina, Johannes Kepler Univ. Linz (Austria); A. Obereder, MathConsult GmbH (Austria); R. Ramlau, Johannes Kepler Univ. Linz (Austria)

$9148 \mathrm{OQ}$ Iterative reconstruction methods in atmospheric tomography: FEWHA, Kaczmarz and Gradient-based algorithm [9148-25]

R. Ramlau, Johannes Kepler Univ. Linz (Austria) and Johann Radon Institute for Computational and Applied Mathematics (Austria); D. Saxenhuber, Johannes Kepler Univ. Linz (Austria); M. Yudytskiy, Johann Radon Institute for Computational and Applied Mathematics (Austria)

9148 OR Fast iterative optimal estimation of turbulence wavefronts with recursive block Toeplitz covariance matrix [9148-26]

R. Conan, The Australian National Univ. (Australia)

SESSION 7 POST-PROCESSING AO DATA I

9148 OS Status of point spread function determination for Keck adaptive optics [9148-27] S. Ragland, W. M. Keck Observatory (United States); L. Jolissaint, Univ. of Applied Sciences Western Switzerland (Switzerland); P. Wizinowich, C. Neyman, W. M. Keck Observatory (United States) 
9148 OU GPI PSF subtraction with TLOCI: the next evolution in exoplanet/disk high-contrast imaging [9148-29]

C. Marois, National Research Council Canada (Canada); C. Correia, National Research Council Canada (Canada) and Univ. do Porto (Portugal); R. Galicher, National Research Council Canada (Canada) and LESIA, Observatoire de Paris, CNRS (France); P. Ingraham, B. Macintosh, Stanford Univ. (United States); T. Currie, Univ. of Toronto (Canada); R. De Rosa, Arizona State Univ. (United States)

\section{SESSION $8 \quad$ AO FOR ELTS}

9148 OW The Giant Magellan Telescope adaptive optics program (Invited Paper) [9148-31] A. H. Bouchez, Giant Magellan Telescope Corp. (United States); D. S. Acton, Ball Aerospace \& Technologies Corp. (United States); R. Biasi, Microgate S.r.I. (Italy); R. Conan, The Australian National Univ. (Australia); B. Espeland, Giant Magellan Telescope Corp. (United States); S. Esposito, INAF - Osservatorio Astrofisico di Arcetri (Italy); J. M. Filgueira, Giant Magellan Telescope Corp. (United States); D. Gallieni, A.D.S. International S.r.I. (Italy); B. A. McLeod, Smithsonian Astrophysical Observatory (United States); E. Pinna, INAF Osservatorio Astrofisico di Arcetri (Italy); F. Santoro, G. Trancho, Giant Magellan Telescope Corp. (United States); M. A. van Dam, Flat Wavefronts (New Zealand)

9148 OX Adaptive optics program at TMT (Invited Paper) [9148-32]

C. Boyer, Thirty Meter Telescope Observatory Corp. (United States); S. Adkins, W. M. Keck Observatory (United States); D. R. Andersen, J. Atwood, National Research Council Canada (Canada); Y. Bo, Technical Institute of Physics and Chemistry (China); P. Byrnes, K. Caputa, National Research Council Canada (Canada); J. Cavaco, AOA Xinetics (United States); B. Ellerbroek, L. Gilles, Thirty Meter Telescope Observatory Corp. (United States); J. Gregory, MIT Lincoln Lab. (United States); G. Herriot, National Research Council Canada (Canada); P. Hickson, The Univ. of British Columbia (Canada); Z. Ljusic, National Research Council Canada (Canada); D. Manter, AOA Xinetics (United States); C. Marois, National Research Council Canada (Canada); A. Otárola, Thirty Meter Telescope Observatory Corp. (United States); H. Pagès, CILAS (France); M. Schoeck, Thirty Meter Telescope Observatory Corp. (United States); J.-C. Sinquin, CILAS (France); M. Smith, P. Spano, K. Szeto, National Research Council Canada (Canada); J. Tang, Institute of Optics and Electronics (China); T. Travouillon, Thirty Meter Telescope Observatory Corp. (United States); J.-P. Véran, National Research Council Canada (Canada); L. Wang, Thirty Meter Telescope Observatory Corp. (United States); K. Wei, Institute of Optics and Electronics (China)

9148 OY Preparing for the phase B of the E-ELT MCAO module project [9148-33]

E. Diolaiti, C. Arcidiacono, G. Bregoli, INAF - Osservatorio Astronomico di Bologna (Italy); R. C. Butler, INAF - Istituto di Astrofisica Spaziale e Fisica Cosmica (Italy); M. Lombini, L. Schreiber, INAF - Osservatorio Astronomico di Bologna (Italy); A. Baruffolo, INAF Osservatorio Astronomico di Padova (Italy); A. Basden, Durham Univ. (United Kingdom); M. Bellazzini, INAF - Osservatorio Astronomico di Bologna (Italy); E. Cascone, INAF Osservatorio Astronomico di Capodimonte (Italy); P. Ciliegi, INAF - Osservatorio Astronomico di Bologna (Italy); F. Cortecchia, INAF - Osservatorio Astronomico di Capodimonte (Italy); G. Cosentino, Univ. degli Studi di Bologna (Italy); V. De Caprio, INAF Osservatorio Astronomico di Capodimonte (Italy); A. De Rosa, INAF - Istituto di Astrofisica Spaziale e Fisica Cosmica (Italy); N. Dipper, Durham Univ. (United Kingdom); S. Esposito, INAF - Osservatorio Astrofisico di Arcetri (Italy); I. Foppiani, INAF - Osservatorio Astronomico di Bologna (Italy); E. Giro, INAF - Osservatorio Astronomico di Padova (Italy); G. Morgante, 
INAF -Istituto di Astrofisica Spaziale e Fisica Cosmica (Italy); R. Myers, Durham Univ. (United Kingdom); F. Patru, INAF - Osservatorio Astrofisico di Arcetri (Italy); R. Ragazzoni, INAF Osservatorio Astronomico di Padova (Italy); A. Riccardi, INAF - Osservatorio Astrofisico di Arcetri (Italy); M. Riva, INAF - Osservatorio Astronomico di Brera (Italy); F. M. Zerbi, INAF Istituto Nazionale di Astrofisica (Italy); M. Casali, B. Delabre, N. Hubin, F. Kerber, M. Le Lovarn, E. Marchetti, S. Ramsay, S. Stroebele, E. Vernet, European Southern Observatory (Germany)

$91480 Z$ Overview of the MICADO SCAO system [9148-34]

Y. Clénet, T. Buey, G. Rousset, LESIA, Observatoire de Paris, CNRS, Univ. Paris Dideroł (France); M. Cohen, GEPI, Observatoire de Paris, CNRS, Univ. Paris Diderot (France); P. Feautrier, IPAG, Univ. Joseph Fourier, CNRS (France); E. Gendron, Z. Hubert, LESIA, Observatoire de Paris, CNRS, Univ. Paris Diderot (France); F. Chemla, GEPI, Observatoire de Paris, CNRS, Univ. Paris Diderot (France); D. Gratadour, P. Baudoz, S. Lacour, A. Boccaletti, A. Sevin, F. Vidal, R. Galicher, D. Perret, B. Le Ruyet, F. Chapron, LESIA, Observatoire de Paris, CNRS, Univ. Paris Diderot (France); E. Stadler, P. Rabou, L. Jocou, S. Rochat, G. Chauvin, IPAG, Univ. Joseph Fourier, CNRS (France); R. Davies, Max-Planck-Institut für extraterrestrische Physik (Germany)

914810 NFIRAOS: first facility AO system for the Thirty Meter Telescope [9148-35]

G. Herriot, D. Andersen, J. Atwood, National Research Council Canada (Canada); C. Boyer, Thirty Meter Telescope Observatory Corp. (United States); P. Byrnes, K. Caputa, National Research Council Canada (Canada); B. Ellerbroek, L. Gilles, Thirty Meter Telescope Observatory Corp. (United States); A. Hill, Z. Ljusic, J. Pazder, M. Rosensteiner, M. Smith, P. Spano, K. Szeto, J.-P. Véran, I. Wevers, National Research Council Canada (Canada); L. Wang, Thirty Meter Telescope Observatory Corp. (United States); R. Wooff, National Research Council Canada (Canada)

\section{SESSION 9 NEW PROPOSED AO SYSTEMS AND CONCEPTS FOR LARGE TELESCOPES AND ELTS}

914811 Pushing the limits of NGSs solely AO: GMCAO and beyond (Invited Paper) [9148-36] R. Ragazzoni, INAF - Osservatorio Astronomico di Padova (Italy)

914812 Second generation Robo-AO instruments and systems [9148-37]

C. Baranec, Institute for Astronomy, Univ. of Hawai'i (United States); R. Riddle, California Institute of Technology (United States); N. M. Law, The Univ. of North Carolina at Chapel Hill (United States); M. R. Chun, J. R. Lu, M. S. Connelley, D. Hall, D. Atkinson, S. Jacobson, Institute for Astronomy, Univ. of Hawaili (United States)

914813 Wide field adaptive optics correction for the GMT using natural guide stars [9148-38] M. A. van Dam, Flat Wavefronts (New Zealand); A. H. Bouchez, Giant Magellan Telescope Corp. (United States); B. A. McLeod, Smithsonian Astrophysical Observatory (United States)

914814 TMT-AGE: wide field of regard multi-object adaptive optics for TMT [9148-39] M. Akiyama, Tohoku Univ. (Japan); S. Oya, Subaru Telescope, National Astronomical Observatory of Japan (United States); Y. H. Ono, Tohoku Univ. (Japan) and Subaru Telescope, National Astronomical Observatory of Japan (United States); H. Takami, S. Ozaki, National Astronomical Observatory of Japan (Japan); Y. Hayano, I. Iwata, Subaru Telescope, National Astronomical Observatory of Japan (United States); K. Hane, T. Wu, Tohoku Univ. (Japan); T. Yamamuro, OptCraft (Japan); Y. Ikeda, Photocoding, Inc. (Japan) 
914816 Review of the latest developments in fast low noise detectors for wavefront sensing in the visible (Invited Paper) [9148-41]

S. M. Adkins, W. M. Keck Observatory (United States)

914817 SAPHIRA detector for infrared wavefront sensing (Invited Paper) [9148-42]

G. Finger, European Southern Observatory (Germany); I. Baker, SELEX ES Ltd. (United Kingdom); D. Alvarez, D. Ives, L. Mehrgan, M. Meyer, J. Stegmeier, European Southern Observatory (Germany); H. J. Weller, SELEX ES Ltd. (United Kingdom)

914818 Revolutionary visible and infrared sensor detectors for the most advanced astronomical AO systems [9148-43]

P. Feautrier, Institut de Planétologie et d'Astrophysique de Grenoble, CNRS, Univ. Grenoble Alpes (France) and First Light Imaging S.A.S. (France); J.-L. Gach, First Light Imaging SAS (France) and Lab. d'Astrophysique de Marseille (France); S. Guieu, Institut de Planétologie et d'Astrophysique de Grenoble, CNRS, Univ. Grenoble Alpes (France); M. Downing, European Southern Observatory (Germany); P. Jorden, e2v technologies Ltd. (United Kingdom); J. Rothman, E. de Borniol, CEA-Leti (France); P. Balard, First Light Imaging S.A.S. (France) and Lab. d'Astrophysique de Marseille, CNRS, Technopôle de Château-Gombert (France); E. Stadler, Institut de Planétologie et d'Astrophysique de Grenoble, CNRS, Univ. Grenoble Alpes (France) and First Light Imaging S.A.S. (France); C. Guillaume, Observatoire de Haute-Provence, CNRS (France); D. Boutolleau, First Light Imaging S.A.S. (France); J. Coussement, SOFRADIR (France); J. Kolb, N. Hubin, European Southern Observatory (Germany); S. Derelle, C. Robert, ONERA (France); J. Tanchon, T. Trollier, A. Ravex, Absolut Systems S.A.S. (France); G. Zins, P. Kern, T. Moulin, S. Rochat, A. Delpoulbé, J.-B. Lebouqun, Institut de Planétologie et d'Astrophysique de Grenoble, CNRS, Univ. Grenoble Alpes (France)

914819 OCAM2S: an integral shutter ultrafast and low noise wavefront sensor camera for laser guide stars adaptive optics systems [9148-44]

J.-L. Gach, Lab. d'Astrophysique de Marseille, CNRS, Aix Marseille Univ. (France) and First Light Imaging S.A.S. (France); P. Feautrier, Institut de Planétologie et d'Astrophysique de Grenoble, CNRS (France) and First Light Imaging S.A.S. (France); P. Balard, Lab. d'Astrophysique de Marseille, CNRS, Aix Marseille Univ. (France) and First Light Imaging S.A.S. (France); C. Guillaume, Observatoire de Haute-Provence, CNRS (France) and First Light Imaging S.A.S. (France); E. Stadler, Institut de Planétologie et d'Astrophysique de Grenoble, CNRS (France) and First Light Imaging S.A.S. (France)

$91481 \mathrm{~A} \quad$ Very low noise Shack-Hartmann wavefront sensor for adaptive optics in the near-IR [9148-45]

S. Gousset, C. Robert, ONERA (France); T. Fusco, ONERA (France) and Lab. d'Astrophysique de Marseille, CNRS, Univ. d'Aix-Marseille (France); V. Michau, C. Petit, S. Derelle, J. Deschamps, ONERA (France); P. Feautrier, E. Stadler, Institut de Planétologie et d'Astrophysique de Grenoble, CNRS (France); E. D. de Borniol, J. Rothman, MINATEC (France); J. Coussement, SOFRADIR (France) 
9148 1B Status of the ARGOS project (Invited Paper) [9148-46]

S. Rabien, L. Barl, Max-Planck-Institut für extraterrestrische Physik (Germany); U. Beckmann, Max-Planck-Institut für Radioastronomie (Germany); M. Bonaglia, INAF - Osservatorio Astrofisico di Arcetri (Italy); J. L. Borelli, Max-Planck-Institut für Astronomie (Germany); J. Brynnel, Large Binocular Telescope Observatory (United States); P. Buschkamp, MaxPlanck-Institut für extraterrestrische Physik (Germany); L. Busoni, INAF - Osservatorio Astrofisico di Arcetri (Italy); J. Christou, Large Binocular Telescope Observatory (United States); C. Connot, Max-Planck-Institut für Radioastronomie (Germany); R. Davies, M. Deysenroth, Max-Planck-Institut für extraterrestrische Physik (Germany); S. Esposito, INAF - Osservatorio Astrofisico di Arcetri (Italy); W. Gässler, Max-Planck-Institut für Astronomie (Germany); H. Gemperlein, Max-Planck-Institut für extraterrestrische Physik (Germany); M. Hart, The Univ. of Arizona (United States); M. Kulas, Max-Planck-Institut für Astronomie (Germany); M. Lefebvre, Large Binocular Telescope Observatory (United States); M. Lehmitz, Max-Planck-Institut für Astronomie (Germany); T. Mazzoni, INAF - Osservatorio Astrofisico di Arcetri (Italy); E. Nussbaum, Max-Planck-Institut für Radioastronomie (Germany); G. Orban de Xivry, Max-Planck-Institut für extraterrestrische Physik (Germany); D. Peter, Max-Planck-Institut für Astronomie (Germany); A. Quirrenbach, Landessternwarte Heidelberg (Germany); W. Raab, Max-Planck-Institut für extraterrestrische Physik (Germany); G. Rahmer, Large Binocular Telescope Observatory (United States); J. Storm, Leibniz-Institut für Astrophysik Połsdam (Germany); J. Ziegleder, Max-Planck-Institut für extraterrestrische Physik (Germany)

9148 IC Laser guidestar uplink correction using a MEMS deformable mirror: on-sky test results and implications for future AO systems [9148-47]

A. P. Norton, D. T. Gavel, UC Observatory Lab. For Adaptive Optics (United States); M. Helmbrecht, C. Kempf, Iris AO, Inc. (United States); E. Gates, K. Chloros, D. Redel, Lick Observatory (United States); R. Kupke, D. Dillon, UC Observatory Lab. For Adaptive Optics (United States)

9148 1D PULSE: The Palomar Ultraviolet Laser for the Study of Exoplanets [9148-126]

C. Baranec, Institute for Astronomy, Univ. of Hawaili (United States); R. G. Dekany, California Institute of Technology (United States); R. S. Burruss, Jet Propulsion Lab. (United States); B. P. Bowler, California Institute of Technology (United States); M. van Dam, Flat Wavefronts (New Zealand); R. Riddle, California Institute of Technology (United States); J. C. Shelton, T. Truong, J. Roberts, Jet Propulsion Lab. (United States); J. Milburn, California Institute of Technology (United States); J. Tesch, Jet Propulsion Lab. (United States)

\section{SESSION 12 PATHFINDERS TO ENABLE AO ON ELTS AND NEW AO CONCEPTS I}

9148 IF Adaptive optics for space debris tracking (Invited Paper) [9148-51]

F. Bennet, C. D'Orgeville, The Australian National Univ. (Australia); Y. Gao, EOS Space Systems Pty. Ltd. (Australia); W. Gardhouse, N. Paulin, I. Price, F. Rigaut, The Australian National Univ. (Australia); I. Ritchie, C. Smith, EOS Space Systems Pty. Ltd. (Australia); K. Uhlendorf, The Australian National Univ. (Australia); Y. Wang, EOS Space Systems Pty. Ltd. (Australia) 
9148 IG Multi-object adaptive optics on-sky results with RAVEN (Invited Paper) [9148-50]

O. Lardière, Univ. of Victoria (Canada); D. Andersen, NRC - Herzburg Institute of Astrophysics (Canada); C. Blain, C. Bradley, D. Gamroth, K. Jackson, P. Lach, R. Nash, K. Venn, Univ. of Victoria (Canada); J.-P. Véran, National Research Council Canada (Canada); C. Correia, Univ. do Porto (Portugal); S. Oya, Y. Hayano, H. Terada, Subaru Telescope, National Astronomical Observatory of Japan (United States); Y. Ono, Subaru Telescope, National Astronomical Observatory of Japan (United States) and Tohoku Univ. (Japan); M. Akiyama, Tohoku Univ. (Japan)

\section{SESSION 13 PATHFINDERS TO ENABLE AO ON ELTS AND NEW AO CONCEPTS II}

$914811 \quad$ CANARY phase B: on-sky open-loop tomographic LGS AO results [9148-52]

T. Morris, Durham Univ. (United Kingdom); E. Gendron, LESIA, CNRS, Observatoire de Paris (France); A. Basden, Durham Univ. (United Kingdom); O. Martin, LESIA, CNRS, Observatoire de Paris (France); J. Osborn, Durham Univ. (United Kingdom); D. Henry, UK Astronomy Technology Ctr. (United Kingdom); Z. Hubert, LESIA, CNRS, Observatoire de Paris (France); G. Sivo, Lab. Charles Fabry, CNRS, Institut d'Optique Graduate School (France) and ONERA (France); D. Gratadour, F. Chemla, A. Sevin, M. Cohen, LESIA, CNRS, Observatoire de Paris (France); E. Younger, Durham Univ. (United Kingdom); F. Vidal, LESIA, CNRS, Observatoire de Paris (France); R. Wilson, T. Butterley, U. Bitenc, A. Reeves, N. Bharmal, Durham Univ. (United Kingdom); H.-F. Raynaud, C. Kulcsar, Lab. Charles Fabry, CNRS, Institut d'Optique Graduate School (France); J.-M. Conan, ONERA (France); J.-M. Huet, D. Perret, LESIA, CNRS, Observatoire de Paris (France); C. Dickson, D. Atkinson, T. Bailie, A. Longmore, S. Todd, UK Astronomy Technology Ctr. (United Kingdom); G. Talbot, S. Morris, Durham Univ. (United Kingdom); G. Rousset, LESIA, CNRS, Observatoire de Paris (France); R. Myers, Durham Univ. (United Kingdom)

$91481 \mathrm{~J}$ A measurement of the systematic astrometric error in GeMS and the short-term astrometric precision in ShaneAO [9148-53]

S. M. Ammons, Lawrence Livermore National Lab. (United States); B. Neichel, Lab. d'Astrophysique de Marseille, CNRS, Aix-Marseille Univ. (France) and Gemini Observatory (Chile); J. Lu, Institute for Astronomy, Univ. of Hawaili (United States); D. T. Gavel, S. Srinath, R. McGurk, A. Rudy, C. Rockosi, Univ. of California, Santa Cruz (United States); C. Marois, National Research Council Canada (Canada); B. Macintosh, Stanford Univ. (United States); D. Savransky, Cornell Univ. (United States); R. Galicher, LESIA, CNRS, Observatoire de Paris (France); E. Bendek, NASA Ames Research Ctr. (United States); O. Guyon, The Univ. of Arizona (United States); E. Marin, V. Garrel, G. Sivo, Gemini Observatory (Chile)

9148 1K imaka: a path-finder ground-layer adaptive optics system for the University of Hawaii 2.2meter telescope on Maunakea [9148-54] M. R. Chun, Institute for Astronomy, Univ. of Hawai'i (United States); O. Lai, Gemini Observatory (United States) and Subaru Telescope, National Astronomical Observatory of Japan (United States); D. Toomey, Mauna Kea Infrared LLC (United States); J. R. Lu, C. Baranec, Institute for Astronomy, Univ. of Hawai'i (United States); S. Thibault, D. Brousseau, Univ. Laval (Canada); H. Zhang, ImmerVision (Canada); Y. Hayano, S. Oya, Gemini Observatory (United States) 
9148 1L Circumstellar disk and planet imaging with AO (Invited Paper) [9148-55]

M. Janson, Queen's Univ. Belfast (United Kingdom)

$91481 \mathrm{M}$ Into the blue: AO science with MagAO in the visible (Invited Paper) [9148-56]

L. M. Close, J. R. Males, K. B. Follette, P. Hinz, K. Morzinski, Y.-L. Wu, Steward Observatory, The Univ. of Arizona (United States); D. Kopon, Max-Planck-Institut für Astronomie (Germany); A. Riccardi, S. Esposito, A. Puglisi, E. Pinna, M. Xompero, R. Briguglio, F. Quiros-Pacheco, INAF - Osservatorio Astrofisico di Arcetri (Italy)

914810 Results from the Gemini NICI Planet-Finding Campaign (Invited Paper) [9148-58]

B. A. Biller, The Univ. of Edinburgh (United Kingdom); M. C. Liu, Institute for Astronomy, Univ. of Hawai'i (United States); Z. Wahhaj, European Southern Observatory (Chile); E. L. Nielsen, Institute for Astronomy, Univ. of Hawai'i (United States); T. L. Hayward, Gemini Observatory (Chile); M. R. Chun, Institute for Astronomy, Univ. of Hawaili (United States); L. M. Close, Steward Observatory, The Univ. of Arizona (United States); C. Ftaclas, Institute for Astronomy, Univ. of Hawai'i (United States); J. R. Males, Steward Observatory, The Univ. of Arizona (United States); M. Hartung, Gemini Observatory (Chile); I. N. Reid, Space Telescope Science Institute (United States); E. Shkolnik, Lowell Observatory (United States); A. J. Skemer, Steward Observatory, The Univ. of Arizona (United States); M. Tecza, N. A. Thatte, F. Clarke, Univ. of Oxford (United Kingdom); D. Toomey, Mauna Kea Infrared LLC (United States)

\section{SESSION 15 STATUS OF CURRENT AO INSTRUMENT PROJECTS II}

9148 IR Adaptive optics at the Subaru telescope: current capabilities and development (Invited Paper) [9148-60]

O. Guyon, Y. Hayano, Subaru Telescope, National Astronomical Observatory of Japan

(United States); M. Tamura, National Astronomical Observatory of Japan (Japan); T. Kudo, S. Oya, Y. Minowa, O. Lai, N. Jovanovic, N. Takato, Subaru Telescope, National Astronomical Observatory of Japan (United States); J. Kasdin, T. Groff, Princeton Univ. (United States); M. Hayashi, National Astronomical Observatory of Japan (Japan); N. Arimoto, Subaru Telescope, National Astronomical Observatory of Japan (United States); H. Takami, National Astronomical Observatory of Japan (Japan); C. Bradley, Univ. of Victoria (Canada); H. Sugai, Kavli Institute for the Physics and Mathematics of the Universe, The Univ. of Tokyo (Japan); G. Perrin, LESIA, CNRS, Observatoire de Paris (France); P. Tuthill, The Univ. of Sydney (Australia); B. Mazin, Univ. of California, Santa Barbara (United States)

9148 is Solar adaptive optics with the DKIST: status report [9148-61]

L. C. Johnson, K. Cummings, M. Drobilek, S. Gregory, S. Hegwer, E. Johansson, J. Marino, K. Richards, T. Rimmele, P. Sekulic, F. Wöger, National Solar Observatory (United States)

9148 IT GREGOR MCAO looking at the Sun [9148-62]

D. Schmidt, National Solar Observatory (United States); T. Berkefeld, F. Heidecke, A. Fischer,

O. von der Lühe, D. Soltau, Kiepenheuer-Institut für Sonnenphysik (Germany) 
$91481 \mathrm{U}$ Final performance and lesson-learned of SAXO, the VLT-SPHERE extreme AO: from early design to on-sky results [9148-63]

T. Fusco, J.-F. Sauvage, ONERA (France) and Lab. d'Astrophysique de Marseille, CNRS, AixMarseille Univ. (France); C. Petit, ONERA (France); A. Costille, K. Dohlen, Lab.

d'Astrophysique de Marseille, CNRS, Aix-Marseille Univ. (France); D. Mouillet, J.-L. Beuzit, Institut de Planétologie et d'Astrophysique de Grenoble, CNRS (France); M. Kasper, M. Suarez, C. Soenke, European Southern Observatory (Germany); E. Fedrigo, LESIA, CNRS, Observatoire de Paris (France); M. Downing, Institut de Planétologie et d'Astrophysique de Grenoble, CNRS (France); P. Baudoz, A. Sevin, D. Perret, LESIA, CNRS, Observatoire de Paris (France); A. Barrufolo, B. Salasnich, INAF - Osservatorio Astronomico di Bologna (Italy); P. Puget, F. Feautrier, S. Rochat, T. Moulin, A. Deboulbé, Institut de Planétologie et d'Astrophysique de Grenoble, CNRS (France); E. Hugot, A. Vigan, Lab. d'Astrophysique de Marseille, CNRS, Aix-Marseille, Univ. (France); D. Mawet, J. Girard, N. Hubin, European Southern Observatory (Germany)

9148 IV Gender equity issues in astronomy: facts, fiction, and what the adaptive optics community can do to close the gap (Invited Paper) [9148-64]

C. D'Orgeville, F. Rigaut, The Australian National Univ. (Australia); S. Maddison, Swinburne Univ. of Technology (Australia); E. Masciadri, INAF - Osservatorio Astrofisico di Arcetri (Italy)

\section{SESSION 16 CHARACTERIZATION, MEASUREMENT AND MODELING OF THE DISTRURBANCES FACED BY AO}

9148 IW Review on atmospheric turbulence monitoring (Invited Paper) [9148-65] G. Lombardi, J. Navarrete, European Southern Observatory (Chile); M. Sarazin, European Southern Observatory (Germany)

9148 1X Turbulence profiling methods applied to ESO's adaptive optics facility [9148-66] J. Valenzuela, Pontificia Univ. Católica de Chile (Chile) and European Southern Observatory (Germany); C. Béchet, Pontificia Univ. Católica de Chile (Chile); A. GarciaRissmann, F. Gonté, J. Kolb, M. Le Louarn, European Southern Observatory (Germany); B. Neichel, Lab. d'Astrophysique de Marseille, CNRS, Aix Marseille Univ. (France); P.-Y. Madec, European Southern Observatory (Germany); A. Guesalaga, Pontificia Univ. Católica de Chile (Chile)

914812 Progress towards wind predictive control on ShaneAO: test bench results [9148-68] A. R. Rudy, S. Srinath, Univ. of California, Santa Cruz (United States); L. Poyneer, S. M. Ammons, Lawrence Livermore National Lab. (United States); D. Gavel, R. Kupke, D. Dillon, C. Rockosi, Univ. of California Observatories (United States)

\section{SESSION 17 EXTREME AO II}

914820 Direct imaging of exoplanets in the habitable zone with adaptive optics (Invited Paper) [9148-69]

J. R. Males, L. M. Close, O. Guyon, K. Morzinski, Steward Observatory, The Univ. of Arizona (United States); A. Puglisi, INAF - Osservatorio Astrofisico di Arcetri (Italy); P. Hinz, K. B. Follette, Steward Observatory, The Univ. of Arizona (United States); J. D. Monnier, Univ. of Michigan (United States); V. Tolls, Harvard-Smithsonian Ctr. for Astrophysics (United States); T. J. Rodigas, A. Weinberger, A. Boss, Carnegie Institution of Washington 
(United States); D. Kopon, Max-Planck-Institut für Astronomie (Germany); Y. Wu, Steward Observatory, The Univ. of Arizona (United States); S. Esposito, A. Riccardi, M. Xompero, R. Briguglio, E. Pinna, INAF - Osservatorio Astrofisico di Arcetri (Italy)

914821 On-sky speckle nulling with the Subaru Coronagraphic Extreme AO (SCExAO) instrument [9148-70]

F. Martinache, Lab. J.L. Lagrange, CNRS, Observatorie de la Côte d'Azur (France) and Subaru Telescope, National Astronomical Observatory of Japan (United States); O. Guyon, Subaru Telescope, National Astronomical Observatory of Japan (United States) and The Univ. of Arizona (United States); N. Jovanovic, C. Clergeon, G. Singh, T. Kudo, Subaru Telescope, National Astronomical Observatory of Japan (United States)

914822 Real-time speckle sensing and suppression with project 1640 at Palomar [9148-71]

G. Vasisht, E. Cady, C. Zhai, T. Lockhart, Jet Propulsion Lab. (United States);

B. Oppenheimer, American Museum of Natural History (United States)

\section{Part Two}

\section{SESSION 18 WAVEFRONT CORRECTION II}

914824 On the way to build the M4 Unit for the E-ELT [9148-73]

E. Vernet, M. Cayrel, N. Hubin, European Southern Observatory (Germany); R. Biasi, Microgate S.r.I. (Italy); D. Gallieni, M. Tintori, A.D.S. International S.r.I. (Italy)

914825 Recent improvements of high density magnetic deformable mirrors: faster, larger and stronger [9148-74]

J. Charton, ALPAO S.A.S. (France); U. Bitenc, Durham Univ. (United Kingdom); J.-F. Curis,

S. Camet, R. Di Chiaro, R. Bougeard, ALPAO S.A.S. (France)

\section{SESSION 19 STATUS OF CURRENT AO INSTRUMENT PROJECTS III}

914826 The ERIS adaptive optics system [9148-75]

E. Marchetti, E. Fedrigo, M. Le Louarn, P.-Y. Madec, C. Soenke, R. Brast, R. Conzelmann,

B. Delabre, M. Duchateau, C. Frank, B. Klein, P. Amico, N. Hubin, European Southern Observatory (Germany); S. Esposito, J. Antichi, L. Carbonaro, A. Puglisi, F. Quirós-Pacheco, A. Riccardi, M. Xompero, INAF - Osservatorio Astrofisico di Arcetri (Italy)

914827 Status of the PALM-3000 high order adaptive optics instrument [9148-4]

R. S. Burruss, Jet Propulsion Lab. (United States); R. G. Dekany, Caltech Optical Observatories (United States); J. E. Roberts, J. C. Shelton, J. K. Wallace, J. A. Tesch, D. L. Palmer, Jet Propulsion Lab. (United States); D. Hale, Caltech Optical Observatories (United States); R. Bartos, Jet Propulsion Lab. (United States); K. M. Rykoski, C. M. Heffner, Palomar Observatory, California Institute of Technology (United States); J. E. Eriksen, Jet Propulsion Lab. (United States); F. Vescelus, California Institute of Technology (United States) 
914828 Pathfinder first light: alignment, calibration, and commissioning of the LINC-NIRVANA ground-layer adaptive optics subsystem [9148-77]

D. Kopon, A. Conrad, Max-Planck-Institut für Astronomie (Germany); C. Arcidiacono, INAF Osservatorio Astrofisico di Bologna (Italy); T. Herbst, Max-Planck-Institut für Astronomie (Germany); V. Viotto, J. Farinato, M. Bergomi, R. Ragazzoni, L. Marafatto, INAF -

Osservatorio Astronomico di Padova (Italy); H. Baumeister, T. Bertram, J. Berwein, F. Briegel, R. Hofferbert, F. Kittmann, M. Kürster, L. Mohr, K. Radhakrishnan, Max-Planck-Institut für Astronomie (Germany)

\section{SESSION 20 WAVEFRONT SENSING II}

$91482 \mathrm{~A}$ Strategies to cope with sodium layer profile variations in laser guide star AO systems (Invited Paper) [9148-79]

B. L. Ellerbroek, Thirty Meter Telescope Observatory Corp. (United States)

9148 2B A near-infrared tip-tilt sensor for the Keck I laser guide star adaptive optics system [9148-80] P. Wizinowich, W. M. Keck Observatory (United States); R. Smith, California Institute of Technology (United States); R. Biasi, Microgate S.r.I. (Italy); S. Cetre, W. M. Keck Observatory (United States); R. Dekany, California Institute of Technology (United States);

B. Femenia-Castella, W. M. Keck Observatory (United States); J. Fucik, D. Hale, California Institute of Technology (United States); C. Neyman, W. M. Keck Observatory (United States); D. Pescoller, Microgate S.r.l. (Italy); S. Ragland, P. Stomski, W. M. Keck Observatory (United States); M. Andrighettoni, Microgate S.r.l. (Italy); R. Bartos, K. Bui, California Institute of Technology (United States); A. Cooper, W. M. Keck Observatory (United States); J. Cromer, California Institute of Technology (United States); M. van Dam, Flat Wavefronts (New Zealand); M. Hess, E. James, J. Lyke, W. M. Keck Observatory (United States); H. Rodriguez, California Institute of Technology (United States); T. Stalcup, W. M. Keck Observatory (United States)

$91482 \mathrm{E} \quad$ A miniature curvature wavefront sensor with coherent fiber image bundle [9148-83] J. Zheng, Australian Astronomical Observatory (Australia); S. Richards, Australian Astronomical Observatory (Australia) and The Univ. of Sydney (Australia); M. Goodwin, J. Lawrence, Australian Astronomical Observatory (Australia); S. Leon-Saval, A. Argyros, The Univ. of Sydney (Australia)

\section{SESSION 21 ADVANCES IN AO CONTROL II}

9148 2F Results of the NFIRAOS RTC trade study (Invited Paper) [9148-84] J.-P. Véran, National Research Council Canada (Canada); C. Boyer, B. L. Ellerbroek, L. Gilles, Thirty Meter Telescope Observatory Corp. (United States); G. Herriot, D. A. Kerley, Z. Ljusic, National Research Council Canada (Canada); E. A. McVeigh, R. Prior, Univ. of Victoria (Canada); M. Smith, National Research Council Canada (Canada); L. Wang, Thirty Meter Telescope Observatory Corp. (United States)

91482 Enabling technologies for GPU driven adaptive optics real-time control [9148-85] A. Sevin, D. Perret, D. Gratadour, M. Lainé, J. Brulé, B. Le Ruyet, LESIA, CNRS, Univ. Paris Diderot (France) 
9148 2J Physical optics modeling of sky coverage for TMT NFIRAOS with advanced LQG controller [9148-88]

L. Wang, L. Gilles, B. Ellerbroek, Thirty Meter Telescope Observatory Corp. (United States);

C. Correia, Univ. do Porto (Portugal)

$91482 \mathrm{~K}$ Tomography and calibration for Raven: from simulations to laboratory results [9148-89] K. Jackson, Univ. of Victoria (Canada); C. Correia, Univ. do Porto (Portugal); O. Lardière, Univ. of Victoria (Canada); D. Andersen, NRC - Herzburg Institute of Astrophysics (Canada); C. Bradley, L. Pham, C. Blain, R. Nash, D. Gamroth, Univ. of Victoria (Canada); J.-P. Véran, NRC - Herzburg Institute of Astrophysics (Canada)

$91482 \mathrm{~L} \quad$ Thirty Meter Telescope astrometry error budget [9148-90]

M. Schöck, Thirty Meter Telescope Observatory Corp. (United States); T. Do, Univ. of Toronto (Canada); B. Ellerbroek, L. Gilles, Thirty Meter Telescope Observatory Corp. (United States); G. Herriot, National Research Council Canada (Canada); L. Meyer, Univ. of California, Los Angeles (United States); R. Suzuki, National Astronomical Observatory of Japan (Japan); L. Wang, Thirty Meter Telescope Observatory Corp. (United States); S. Yelda, National Astronomical Observatory of Japan (Japan)

9148 2M Design and numerical simulations of the GMT Natural Guide star WFS [9148-91]

E. Pinna, G. Agapito, F. Quirós-Pacheco, J. Antichi, L. Carbonaro, R. Briguglio, M. Bonaglia,

A. Riccardi, A. Puglisi, V. Biliotti, INAF - Osservatorio Astrofisico di Arcetri (Italy);

C. Arcidiacono, INAF - Osservatorio Astrofisico di Arcetri (Italy) and INAF - Osservatorio Astronomico di Bologna (Italy); M. Xompero, INAF - Osservatorio Astrofisico di Arcetri (Italy); G. Di Rico, A. Valentini, INAF - Osservatorio Astronomico di Teramo (Italy); A. Bouchez, F. Santoro, G. Trancho, Giant Magellan Telescope Corp. (United States); S. Esposito, INAF Osservatorio Astrofisico di Arcetri (Italy)

\section{SESSION 23 POST-PROCESSING AO DATA II}

9148 2N Detailed analysis of the Canary on-sky results at the WHT using Rayleigh laser guide stars [9148-92]

O. Martin, E. Gendron, LESIA, CNRS, Univ. Paris Diderot (France); T. Morris, A. Basden, Durham Univ. (United Kingdom); Z. Hubert, D. Gratadour, LESIA, CNRS, Univ. Paris Diderot (France); J. Osborn, Durham Univ. (United Kingdom); F. Vidal, LESIA, CNRS, Univ. Paris Diderot (France); F. Chemla, GEPI, CNRS, Univ. Paris Diderot (France); G. Rousset, LESIA, CNRS, Univ. Paris Diderot (France); R. Myers, Durham Univ. (United Kingdom)

$914820 \quad$ Image restoration with spatially variable PSF [9148-182]

P. Ciliegi, INAF - Osservatorio Astronomico di Bologna (Italy); A. La Camera, Univ. degli Studi di Genova (Italy); L. Schreiber, M. Bellazzini, INAF - Osservatorio Astronomico di Bologna (Italy); M. Bertero, P. Boccacci, Univ. degli Studi di Genova (Italy); E. Diolaiti, I. Foppiani, M. Lombini, INAF - Osservatorio Astronomico di Bologna (Italy); D. Massari, Univ. degli Studi di Bologna (Italy); P. Montegriffo, INAF - Osservatorio Astronomico di Bologna (Italy); M. Talia, Univ. degli Studi di Bologna (Italy)

9148 2P Perspectives on phase rertrieval and phase diversity in astronomy [9148-94]

R. A. Gonsalves, Tufts Univ. (United States) 
$91482 R \quad$ Ground layer correction: the heart of LINC-NIRVANA [9148-97]

K. K. Radhakrishnan Santhakumari, Max-Planck-Institut für Astronomie (Germany); L. Marafatto, INAF - Osservatorio Astronomico di Padova (Italy) and Univ. degli Studi di Padova (Italy); M. Bergomi, V. Viotto, J. Farinato, R. Ragazzoni, INAF - Osservatorio Astronomico di Padova (Italy); T. Herbst, T. Bertram, Max-Planck-Institut für Astronomie (Germany); M. Dima, INAF - Osservatorio Astronomico di Padova (Italy); P. Bizenberger, F. Briegel, F. Kittmann, L. Mohr, Max-Planck-Institut für Astronomie (Germany); D. Magrin, INAF - Osservatorio Astronomico di Padova (Italy)

$91482 S \quad$ ULTIMATE-SUBARU: project status [9148-98]

Y. Hayano, Subaru Telescope, National Astronomical Observatory of Japan (United States); M. Akiyama, Tohoku Univ. (Japan); T. Hattori, I. Iwata, Subaru Telescope, National Astronomical Observatory of Japan (United States); T. Kodama, National Astronomical Observatory of Japan (Japan); O. Lai, Subaru Telescope, National Astronomical Observatory of Japan (United States) and Gemini Observatory (United States); Y. Minowa, Subaru Telescope, National Astronomical Observatory of Japan (United States); Y. Ono, Tohoku Univ. (Japan); S. Oya, K. Takiura, I. Tanaka, Y. Tanaka, N. Arimito, Subaru Telescope, National Astronomical Observatory of Japan (United States)

9148 2T Integration and bench testing for the GRAVITY Coudé IR adaptive optics (CIAO) wavefront sensor [9148-99] C. Deen, Max-Planck-Institut für Astronomie (Germany); P. Yang, Max-Planck-Institut für Astronomie (Germany) and Shanghai Institute of Optics and Fine Mechanics (China); A. Huber, Max-Planck-Institut für Astronomie (Germany); M. Suarez-Valles, European Southern Observatory (Germany); S. Hippler, W. Brandner, Max-Planck-Institut für Astronomie (Germany); E. Gendron, Y. Clénet, LESIA, CNRS, Observatoire de Paris, Univ. Paris Diderot (France); S. Kendrew, Max-Planck-Institut für Astronomie (Germany) and Univ. of Oxford (United Kingdom); A. Glauser, Max-Planck-Institut für Astronomie (Germany) and ETH Zürich (Switzerland); R. Klein, W. Laun, R. Lenzen, U. Neumann, J. Panduro, J. Ramos, R.-R. Rohloff, A. Salzinger, N. Zimmerman, T. Henning, Max-Planck-Institut für Astronomie (Germany); K. Perraut, UJF-Grenoble 1, Institut de Planétologie et d'Astrophysique de Grenoble, CNRS (France); G. Perrin, LESIA, CNRS, Observatoire de Paris, Univ. Paris Diderot (France) and Groupement d'Intérêt Scientifique PHASE (France); C. Straubmeier, Univ. zu Köln (Germany); A. Amorim, Lab. de Sistemas, Instrumentação e Modelação em Ciências e Tecnologias do Ambiente e do Espaço (Portugal); F. Eisenhaver, Max-Planck-Institut für extraterrestrische Physik (Germany)

$91482 \mathrm{U}$ The multi-conjugate adaptive optics system of the New Solar Telescope at Big Bear Solar Observatory [9148-100]

D. Schmidt, National Solar Observatory (United States); N. Gorceix, X. Zhang, Big Bear Solar Observatory (United States); J. Marino, National Solar Observatory (United States);

R. Coulter, S. Shumko, P. Goode, Big Bear Solar Observatory (United States); T. Rimmele, National Solar Observatory (United States); T. Berkefeld, Kiepenhever-Institut für Sonnenphysik (Germany)

$91482 \mathrm{~V}$ GALACSI integration and functional tests [9148-101]

P. La Penna, S. Ströbele, E. Aller Carpentier, J. Argomedo, R. Arsenault, R. D. Conzelmann,

B. Delabre, R. Donaldson, M. Duchateau, E. Fedrigo, F. Gago, N. Hubin, J. Quentin,

P. Jolley, M. Kiekebusch, J. P. Kirchbauer, B. Klein, J. Kolb, H. Kuntschner, M. Le Louarn, 
J. L. Lizon, P.-Y. Madec, A. Manescau, L. Mehrgan, B. Sedghi, M. Suárez Valles, C. Soenke, S. Tordo, J. Vernet, S. Zampieri, European Southern Observatory (Germany)

$91482 \mathrm{~W}$ The first portable solar and stellar adaptive optics [9148-102]

D. Ren, California State Univ., Northridge (United States) and Nanjing Institute of Astronomical Optics \& Technology (China); R. Li, Nanjing Institute of Astronomical Optics \& Technology (China) and Univ. of Chinese Academy of Sciences (China); X. Zhang, J. Dou, Y. Zhu, G. Zhao, Z. Wu, R. Chen, Nanjing Institute of Astronomical Optics \& Technology (China); C. Liu, Nanjing Institute of Astronomical Optics \& Technology (China) and Univ. of Chinese Academy of Sciences (Central African Republic); F. Yang, C. Yang, Nanjing Institute of Astronomical Optics \& Technology (China) and Univ. of Chinese Academy of Sciences (China)

$91482 Y \quad$ First light of the LINC-NIRVANA Pathfinder experiment [91 48-106] M. Bergomi, V. Viotto, INAF - Osservatorio Astronomico di Padova (Italy); C. Arcidiacono, INAF - Osservatorio Astronomico di Bologna (Italy) and INAF - Osservatorio Astrofisico di Arcetri (Italy); L. Marafatto, INAF - Osservatorio Astronomico di Padova (Italy) and Univ. degli Studi di Padova (Italy); J. Farinato, INAF - Osservatorio Astronomico di Padova (Italy); H. Baumeister, T. Bertram, J. Berwein, F. Briegel, A. Conrad, F. Kittman, D. Kopon,

R. Hofferbert, Max-Planck-Institut für Astronomie (Germany); D. Magrin, INAF - Osservatorio Astronomico di Padova (Italy); K. K. Radhakrishnan Santhakumari, Max-Planck-Institut für Astronomie (Germany); A. Puglisi, M. Xompero, R. Briguglio, F. Quiros-Pacheco, INAF Osservatorio Astrofisico di Arcetri (Italy); T. M. Herbst, Max-Planck-Institut für Astronomie (Germany); R. Ragazzoni, INAF - Osservatorio Astronomico di Padova (Italy)

$91482 Z$ Swimming with ShARCS: comparison of on-sky sensitivity with model predictions for ShaneAO on the Lick Observatory 3-meter telescope [9148-107]

S. Srinath, R. McGurk, C. Rockosi, Univ. of California, Santa Cruz (United States); R. Kupke,

D. Gavel, G. Cabak, D. Cowley, M. Peck, C. Ratliff, E. Gates, D. Dillon, A. Norton,

M. Reining, Univ. of California Observatories (United States)

914830 The CHARA array adaptive optics I: common-path optical and mechanical design, and preliminary on-sky results [9148-108]

X. Che, Univ. of Michigan (United States); L. Sturmann, The CHARA Array, Mountain Wilson Observatory (United States); J. D. Monnier, Univ. of Michigan (United States); T. A. ten Brummelaar, J. Sturmann, The CHARA Array, Mountain Wilson Observatory (United States); S. T. Ridgway, National Optical Astronomy Observatory (United States); M. J. Ireland, Macquaire Univ. (Australia); N. H. Turner, The CHARA Array, Mountain Wilson Observatory (United States); H. A. McAlister, Georgia State Univ. (United States)

914831 Development of a new solar adaptive optics system at the Hida Observatory [9148-109] N. Miura, A. Oh-ishi, S. Aoki, H. Mogaki, S. Kuwamura, Kitami Institute of Technology (Japan); N. Baba, Hokkaido Univ. (Japan); Y. Hanaoka, National Astronomical Observatory of Japan (Japan); M. Yamaguchi, S. Ueno, Y. Nakatani, K. Ichimoto, Kyoto Univ. (Japan)

914833 Optical design of the relay optics for the MICADO SCAO system [9148-111] M. Cohen, F. Chemla, T. Buey, E. Gendron, Z. Hubert, LESIA, CNRS, GEPI Observatoire de Paris (France); M. Hartl, Max-Planck-Institut für extraterrestrische Physik (Germany); Y. Clénet, LESIA, CNRS, GEPI Observatoire de Paris (France); R. Davies, Max-Planck-Institut für extraterrestrische Physik (Germany) 
914834 ARGOS wavefront sensing: from detection to correction [9148-112]

G. Orban de Xivry, Max-Planck-Institut für extraterrestrische Physik (Germany); M. Bonaglia, INAF - Osservatorio Astrofisico di Arcetri (Italy); J. Borelli, Max-Planck-Institut für Astronomie (Germany); L. Busoni, INAF - Osservatorio Astrofisico di Arcetri (Italy); C. Connot, MaxPlanck-Insitut für Radioastronomie (Germany); S. Esposito, INAF - Osservatorio Astrofisico di Arcetri (Italy); W. Gaessler, M. Kulas, Max-Planck-Institut für Astronomie (Germany); T. Mazzoni, A. Puglisi, INAF - Osservatorio Astrofisico di Arcetri (Italy); S. Rabien, Max-PlanckInstitut für extraterrestrische Physik (Germany); J. Storm, Leibniz-Institut für Astrophysik Potsdam (Germany); J. Ziegleder, Max-Planck-Institut für extraterrestrische Physik (Germany)

914835 AO-308: the high-order adaptive optics system at Big Bear Solar Observatory [91 48-113] S. Shumko, N. Gorceix, Big Bear Solar Observatory (United States); S. Choi, Big Bear Solar Observatory (United States) and Korea Astronomy and Space Science Institute (Korea, Republic of); A. Kellerer, Big Bear Solar Observatory (United States); W. Cao, Big Bear Solar Observatory (United States) and New Jersey Institute of Technology (United States); P. R. Goode, New Jersey Institute of Technology (United States); V. Abramenko, Big Bear Solar Observatory (United States); K. Richards, T. R. Rimmele, J. Marino, National Solar Observatory (United States)

914837 Present opto-mechanical design status of NFIRAOS [9148-115] P. W. G. Byrnes, J. Atwood, National Research Council Canada (Canada); M.-A. Boucher, Institut National d'Optique (Canada); J. Fitzsimmons, A. Hill, G. Herriot, P. Spanò, K. Szeto, I. Wevers, National Research Council Canada (Canada)

914838 Altair performance and upgrades [9148-116]

O. Lai, Gemini Observatory (United States) and Subaru Telescope, National Astronomical Observatory of Japan (United States); J.-P. Véran, G. Herriot, NRC - Herzburg Institute of Astrophysics (Canada); J. White, J. Ball, C. Trujillo, Gemini Observatory (United States)

914839 KAPAO first light: the design, construction and operation of a low-cost natural guide star adaptive optics system [9148-117]

S. A. Severson, Sonoma State Univ. (United States); P. I. Choi, Pomona College (United States); K. E. Badham, Sonoma State Univ. (United States); D. Bolger, D. S. Contreras, Pomona College (United States); B. N. Gilbreth, Sonoma State Univ. (United States); C. Guerrero, Harvey Mudd College (United States); E. Littleton, J. Long, L. P. McGonigle, W. A. Morrison, F. Ortega, A. R. Rudy, J. R. Wong, Pomona College (United States); E. Spjut, Harvey Mudd College (United States); C. Baranec, Institute for Astronomy, Univ. of Hawai'i (United States); R. Riddle, California Institute of Technology (United States)

9148 3A Commissioning ShARCS: the Shane adaptive optics infrared camera-spectrograph for the Lick Observatory Shane 3-m telescope [9148-118]

R. McGurk, C. Rockosi, D. Gavel, R. Kupke, M. Peck, T. Pfister, J. Ward, W. Deich, J. Gates, E. Gates, B. Alcott, D. Cowley, D. Dillon, K. Lanclos, D. Sandford, M. Saylor, S. Srinath, Lick Observatory, Univ. of California, Santa Cruz (United States); J. Weiss, Univ. of California, Los Angeles (United States); A. Norton, Lick Observatory, Univ. of California, Santa Cruz (United States)

9148 3B Opto-mechanical design of ShaneAO: the adaptive optics system for the 3-meter Shane Telescope [9148-119]

C. Ratliff, J. Cabak, D. Gavel, R. Kupke, D. Dillon, E. Gates, W. Deich, J. Ward, D. Cowley, T. Pfister, M. Saylor, Lick Observatory, Univ. of California, Santa Cruz (United States) 
9148 3D The NGS Pyramid wavefront sensor for ERIS [9148-122]

A. Riccardi, J. Antichi, F. Quirós-Pacheco, S. Esposito, L. Carbonaro, G. Agapito, V. Biliotti,

R. Briguglio, G. Di Rico, INAF - Osservatorio Astrofisico di Arcetri (Italy); M. Dolci, INAF -

Osservatorio Astronomico di Teramo (Italy); D. Ferruzzi, E. Pinna, A. Puglisi, M. Xompero,

INAF - Osservatorio Astrofisico di Arcetri (Italy); E. Marchetti, E. Fedrigo, M. Le Louarn,

R. Conzelmann, B. Delabre, P. Amico, N. Hubin, European Southern Observatory

(Germany)

POSTER SESSION: LASER GUIDE STAR SYSTEMS

$91483 \mathrm{E} \quad$ A sodium laser guide star facility for the ANU/EOS space debris tracking adaptive optics demonstrator [9148-124]

C. D'Orgeville, F. Bennet, The Australian National Univ. (Australia); M. Blundell, R. Brister,

A. Chan, M. Dawson, Y. Gao, EOS Space Systems Pty. Ltd. (Australia); N. Paulin, I. Price, F. Rigaut, The Australian National Univ. (Australia); I. Ritchie, EOS Space Systems Pty. Ltd. (Australia); M. Sellars, The Australian National Univ. (Australia); C. Smith, EOS Space Systems Pty. Ltd. (Australia); K. Uhlendorf, Jenoptik Optical Systems GmbH (Germany); Y. Wang, EOS Space Systems Pty. Ltd. (Australia)

$91483 G \quad$ Pulsed laser architecture for enhancing backscatter from sodium [9148-127] T. J. Kane, P. D. Hillman, C. A. Denman, FASORtronics LLC (United States)

91483H ARGOS laser system mechanical design [9148-128] M. Deysenroth, M. Honsberg, H. Gemperlein, J. Ziegleder, W. Raab, S. Rabien, L. Barl, MaxPlanck-Institut für extraterrestrische Physik (Germany); W. Gässler, J. L. Borelli, Max-PlanckInstitut für Astronomie (Germany)

914831 Laboratory validation of a laser shaping system before guide star projection [9148-129] S. Zúñiga, Univ. Técnica Federico Santa María (Chile); C. Béchet, H. González-Núñez, Pontificia Univ. Católica de Chile (Chile); B. Neichel, Lab. d'Astrophysique de Marseille, CNRS, Aix Marseille Univ. (France) and Gemini Observatory Southern Operations Ctr. (Chile); V. Fesquet, V. Garrel, Gemini Observatory Southern Operations Ctr. (Chile); P. Escárate, M. Castro, Univ. Técnica Federico Santa María (Chile); D. Guzmán,

A. Guesalaga, Pontificia Univ. Católica de Chile (Chile)

9148 3J Polarization control optimization of the Gemini South beam transfer optics [9148-130] C. Araujo, C. Moreno, V. Fesquet, V. Garrel, C. Marchant, Gemini Observatory (Chile)

9148 3K The ARGOS laser system: green light for ground layer adaptive optics at the LBT [9148-131] W. Raab, S. Rabien, Max-Planck-Institut für extraterrestrische Physik (Germany); W. Gässler, Max-Planck-Institut für Astronomie (Germany); S. Esposito, INAF - Osservatorio Astrofisico di Arcetri (Italy); L. Barl, Max-Planck-Institut für extraterrestrische Physik (Germany); J. Borelli, Max-Planck-Institut für Astronomie (Germany); M. Daysenroth, H. Gemperlein, Max-PlanckInstitut für extraterrestrische Physik (Germany); M. Kulas, Max-Planck-Institut für Astronomie (Germany); J. Ziegleder, Max-Planck-Institut für extraterrestrische Physik (Germany)

$91483 \mathrm{~L} \quad$ Coupling efficiency measurements for long-pulsed solid sodium laser based on measured sodium profile data [9148-133]

K. Jin, Institute of Optics and Electronics (China) and The Key Lab. on Adaptive Optics (China) and Univ. of Chinese Academy of Sciences (China); K. Wei, Institute of Optics and Electronics (China) and The Key Lab. on Adaptive Optics (China); S. Xie, Y. Bo, J. Zuo, 
Technical Institute of Physics and Chemistry (China); P. Wang, Univ. of Chinese Academy of Sciences (China) and Technical Institute of Physics and Chemistry (China); L. Feng, National Observatory of China (China); X. Xue, Univ. of Science and Technology of China (China); M. Li, Institute of Optics and Electronics (China) and The Key Lab. on Adaptive Optics (China); X. Cheng, Wuhan Institute of Physics and Mathematics (China); C. Cui, Anhui Institute of Optics and Fine Mechanics (China); Y. Shen, Q. Bian, J. Yao, Technical Institute of Physics and Chemistry (China); A. Otárola, Thirty Meter Telescope Observatory Corp. (United States); X. Dai, Institute of Optics and Electronics (China) and The Key Lab. on Adaptive Optics (China) and Univ. of Chinese Academy of Sciences (China); Q. Peng, Technical Institute of Physics and Chemistry (China); C. Rao, Institute of Optics and Electronics (China) and The Key Lab. on Adaptive Optics (China); Z. Xu, Technical Institute of Physics and Chemistry (China); Y. Zhang, Institute of Optics and Electronics (China) and The Key Lab. on Adaptive Optics (China)

$91483 \mathrm{M}$ Proposal for a field experiment of elongated Na LGS wave-front sensing in the perspective of the E-ELT [9148-134]

G. Rousset, D. Gratadour, E. Gendron, T. Buey, LESIA, CNRS, Observatoire de Paris, Univ. Paris Diderot (France); R. Myers, T. Morris, A. Basden, G. Talbot, Durham Univ. (United Kingdom); D. Bonaccini Calia, E. Marchetti, T. Pfrommer, European Southern Observatory (Germany)

$91483 \mathrm{~N} \quad$ Using a deformable mirror to generate custom laser guidestar asterisms: simulation and laboratory results [9148-135]

A. P. Norton, S. Srinath, D. Gavel, R. Kupke, D. Dillon, UC Observatory Lab. for Adaptive Optics (United States)

914830 Assembly and test results of the AOF laser guide star units at ESO [9148-136] W. Hackenberg, D. Bonaccini Calia, B. Buzzoni, M. Comin, C. Dupuy, F. Gago, I. M. Guidolin, R. Guzman, R. Holzloehner, L. Kern, J. P. Kirchbauer, S. Lewis, J.-L. Lizon, S. McLay, T. Pfrommer, M. Quattri, J. Quentin, R. Ridings, European Southern Observatory (Germany)

$91483 P \quad$ Laser guide star pointing camera for ESO LGS Facilities [9148-137] D. Bonaccini Calia, European Southern Observatory (Germany); M. Centrone, F. Pedichini, INAF - Osservatorio Astronomico di Roma (Italy); A. Ricciardi, A. Cerruto, Astrel Instruments (Italy); F. Ambrosino, INAF - Osservatorio Astronomico di Roma (Italy)

9148 3Q Evaluating the compliance of Keck's LGSAO automated aircraft protection system with FAA adopted criteria [9148-138]

P. J. Stomski Jr., R. Campbell, W. M. Keck Observatory (United States); T. W. Murphy Jr., Univ. of California, San Diego (United States)

\section{POSTER SESSION: ASTRONOMY WITH AO}

9148 3T Experimental demonstration of brighter sodium resonant scattering with $1.7 \mathrm{GHz}$ sideband repumping for long pulse laser [9148-132]

L. Li, S. Zhang, W. Hua, H. Wang, Y. Ning, X. Xu, National Univ. of Defense Technology (China) 
$91483 \mathrm{U}$ On the use of asymmetric PSF on NIR images of crowded stellar fields [9148-142] G. Fiorentino, INAF - Osservatorio Astronomico di Bologna (Italy); I. Ferraro, G. Iannicola, INAF - Osservatorio Astronomico di Roma (Italy); G. Bono, INAF - Osservatorio Astronomico di Roma (Italy) and Univ. degli Studi di Roma Tor Vergata (Italy); M. Monelli, Instituto de Astrofísica de Canarias (Spain) and Univ. de la Laguna (Spain); V. Testa, INAF - Osservatorio Astronomico di Roma (Italy); C. Arcidiacono, INAF - Osservatorio Astronomico di Bologna (Italy); M. Faccini, INAF - Osservatorio Astronomico di Roma (Italy); R. Gilmozzi, European Southern Observatory (Germany); M. Xompero, R. Briguglio, INAF - Osservatorio Astronomico di Arcetri (Italy)

9148 3V Photometric performance of LGS MCAO with science-based metrics: first results from Gemini/GeMS observations of Galactic globular clusters [9148-143]

P. Turri, Univ. of Victoria (Canada) and NRC - Herzberg Institute of Astrophysics (Canada); A. W. McConnachie, P. B. Stetson, NRC - Herzberg Institute of Astrophysics (Canada); G. Fiorentino, INAF - Osservatorio Astronomico di Bologna (Italy); D. R. Andersen, NRC Herzberg Institute of Astrophysics (Canada); G. Bono, INAF - Osservatorio Astronomico di Bologna (Italy); J.-P. Véran, NRC - Herzberg Institute of Astrophysics (Canada)

9148 3X L'-band AGPM vector vortex coronagraph's first light on LBTI/LMIRCam [9148-145] D. Defrère, Steward Observatory, The Univ. of Arizona (United States); O. Absil, Univ. de Liège (Belgium); P. Hinz, Steward Observatory, The Univ. of Arizona (United States); J. Kunn, Jet Propulsion Lab. (United States); D. Mawet, European Southern Observatory (Chile); B. Mennesson, Jet Propulsion Lab. (United States); A. Skemer, Steward Observatory, The Univ. of Arizona (United States); K. Wallace, Jet Propulsion Lab. (United States); V. Bailey, E. Downey, Steward Observatory, The Univ. of Arizona (United States); C. Delacroix, Univ. de Liège (Belgium); O. Durney, Steward Observatory, The Univ. of Arizona (United States); P. Forsberg, Uppsala Univ. (Sweden); C. Gomez, S. Habraken, Univ. de Liège (Belgium); W. F. Hoffmann, Steward Observatory, The Univ. of Arizona (United States); M. Karlsson, Uppsala Univ. (Sweden); M. Kenworthy, Leiden Observatory (Netherlands); J. Leisenring, M. Montoya, Steward Observatory, The Univ. of Arizona (United States); L. Pueyo, Space Telescope Science Institute (United States); M. Skrutskie, Univ. of Virginia (United States); J. Surdej, Univ. de Liège (Belgium)

\section{POSTER SESSION: WAVEFRONT CORRECTION}

9148 3Y Double drive modes unimorph deformable mirror with high actuator count for astronomical application [9148-146]

Y. Liu, Univ. Of Science and Technology of China (China); J. Ma, Ningbo Univ. (China);

J. Chen, B. Li, J. Chu, Univ. of Science and Technology of China (China)

914832 Payload characterization for CubeSat demonstration of MEMS deformable mirrors [9148-147]

A. Marinan, K. Cahoy, M. Webber, Massachusetts Institute of Technology (United States);

R. Belikov, E. Bendek, NASA Ames Research Ctr. (United States)

914840 Analysis of the static deformation matching between numerical and experimental data on the voice-coil actuated deformable mirrors [9148-148]

C. Del Vecchio, R. Briguglio, A. Riccardi, M. Xompero, INAF - Osservatorio Astrofisico di Arcetri (Italy) 
914842 A compact adaptive optics system with 3mm narrow-interval deformable mirror [9148-150] M. Li, L. Xue, H. Xian, X. Rao, K. Wei, X. Zhang, S. Chen, A. Zhang, D. Chen, C. Rao, Y. Zhang, Institute of Optics and Electronics (China) and The Key Lab. On Adaptive Optics (China)

914843 Optimization of electrode configuration in surface-parallel actuated deformable mirrors [9148-151]

M. Laslandes, S. Pellegrino, J. Steeves, California Institute of Technology (United States); K. Patterson, Jet Propulsion Lab. (United States)

914844 The 384-channel prototype of DM Electronics for ELT AO systems [9148-152] K. Caputa, J. Atwood, G. Herriot, J.-P. Véran, P. Spanò, National Research Council Canada (Canada); A. Zielinski, Univ. of Victoria (Canada)

914845 The deformable secondary mirror of VLT: final electro-mechanical and optical acceptance test results [9148-153]

R. Briguglio, INAF - Osservatorio Astrofisico di Arcetri (Italy); R. Biasi, Microgate S.r.l. (Italy); M. Xompero, A. Riccardi, INAF - Osservatorio Astrofisico di Arcetri (Italy); M. Andrighettoni, D. Pescoller, G. Angerer, Microgate S.r.l. (Italy); D. Gallieni, A.D.S. International S.r.l. (Italy); E. Vernet, J. Kolb, R. Arsenault, P.-Y. Madec, European Southern Observatory (Germany)

914846 Deformable mirror interferometric analysis for the direct imagery of exoplanets [9148-154] J. Mazoyer, R. Galicher, P. Baudoz, LESIA, CNRS, Observatorie de Paris (France); P. Lanzoni, F. Zamkotsian, Lab. d'Astrophysique de Marseille, CNRS, Aix-Marseille Univ. (France); G. Rousset, LESIA, CNRS, Observatorie de Paris (France)

POSTER SESSION: EXTREME AO

914848 Lyot-based low order wavefront sensor: implementation on the Subaru Coronagraphic Extreme Adaptive Optics System and its laboratory performance [9148-157] G. Singh, Subaru Telescope, National Astronomical Observatory of Japan (United States) and LESIA, CNRS, Observatorie de Paris (France); O. Guyon, Subaru Telescope, National Astronomical Observatory of Japan (United States) and Jet Propulsion Lab. (United States); P. Baudoz, LESIA, CNRS, Observatorie de Paris (France); N. Jovanovich, Subaru Telescope, National Astronomical Observatory of Japan (United States); F. Martinache, Observatoire de la Cote d'Azur, CNRS, Univ. de Nice Sophia-Antipolis (France); T. Kudo, Subaru Telescope, National Astronomical Observatory of Japan (United States); E. Serabyn, J. G. Kuhn, Jet Propulsion Lab. (United States)

914849 CHARIS science: performance simulations for the Subaru Telescope's third-generation of exoplanet imaging instrumentation [9148-158]

T. D. Brandt, Institute for Advanced Study (United States); M. W. McElwain, NASA Goddard Space Flight Ctr. (United States); M. Janson, Queen's Univ. Belfast (United Kingdom); G. R. Knapp, Princeton Univ. (United States); K. Mede, The Univ. of Tokyo (Japan); M. A. Limbach, T. Groff, A. Burrows, J. E. Gunn, Princeton Univ. (United States); O. Guyon, Subaru Telescope, National Astronomical Observatory of Japan (United States) and The Univ. of Arizona (United States); J. Hashimoto, The Univ. of Oklahoma (United States); M. Hayashi, National Astronomical Observatory of Japan (Japan); N. Jovanovic, Subaru Telescope, National Astronomical Observatory of Japan (United States); N. J. Kasdin, Princeton Univ. (United States); M. Kuzuhara, National Astronomical Observatory of Japan (Japan) and Tokyo Institute of Technology (Japan); R. H. Lupton, Princeton Univ. (United 
States); F. Martinache, Subaru Telescope, National Astronomical Observatory of Japan (United States) and Laboratoire Lagrange, CNRS, Observatoire de la Côte d'Azur (France); S. Sorahana, Nagoya Univ. (Japan); D. S. Spiegel, Institute for Advanced Study (United States); N. Takato, Subaru Telescope, National Astronomical Observatory of Japan (United States); M. Tamura, National Astronomical Observatory of Japan (Japan) and Univ. of Tokyo (Japan); E. L. Turner, Princeton Univ. (United States) and The Univ. of Tokyo (Japan); R. Vanderbei, Princeton Univ. (United States); J. Wisniewski, Univ. of Oklahoma (United States)

\section{Part Three}

\section{POSTER SESSION: ADVANCES IN AO CONTROL}

9148 4A A software based de-rotation algorithm concept for the new adaptive optics module (NAOMI) for the auxiliary telescopes of the VLTI [9148-159]

E. Aller-Carpentier, E. Marchetti, R. Dorn, F. Delplancke-Stroebele, E. Fedrigo, M. Le Louarn, N. Hubin, J. Paufique, J. Woillez, European Southern Observatory (Germany)

$91484 \mathrm{~B}$ Evaluation of the Xeon phi processor as a technology for the acceleration of real-time control in high-order adaptive optics systems [9148-160]

D. Barr, UK Astronomy Technology Ctr. (United Kingdom) and Heriot-Watt Univ. (United Kingdom); A. Basden, N. Dipper, Durham Univ. (United Kingdom); N. Schwartz, A. Vick, H. Schnetler, UK Astronomy Technology Ctr. (United Kingdom)

9148 4C Preliminary evaluation and comparison of atmospheric turbulence rejection performance for infinite and receding horizon control in adaptive optics systems [9148-161]

M. V. Konnik, J. De Dona, The Univ. of Newcastle (Australia)

9148 4E Multi-input multi-output identification for control of adaptive optics systems [9148-165] R. Muradore, Univ. degli Studi di Verona (Italy); J. Kolb, L. Pettazzi, E. Marchetti, European Southern Observatory (Germany)

$91484 \mathrm{~F}$ Real-time control for the high order, wide field DRAGON AO test bench [9148-168] A. Basden, N. A. Bharmal, U. Bitenc, N. Dipper, T. Morris, R. Myers, A. Reeves, E. Younger, Durham Univ. (United Kingdom)

9148 4G VLT DSM, the control system of the largest deformable secondary mirror ever manufactured [9148-169]

M. Manetti, Microgate S.r.l. (Italy); M. Morandini, P. Mantegazza, Politecnico di Milano (Italy); R. Biasi, M. Andrighettoni, Microgate S.r.I. (Italy); D. Gallieni, A.D.S. International S.r.I. (Italy)

$91484 \mathrm{H} \quad$ Anti-windup control of tip-tilt mirror [9148-172]

J.-P. Folcher, Lab. Lagrange, CNRS, Univ. de Nice Sophia Antipolis (France)

$91484 \mathrm{~J}$ Woofer-tweeter deformable mirror control for closed-loop adaptive optics: theory and practice [9148-174]

D. Gavel, Univ. of California Observatories (United States); A. Norton, Lockheed Martin Space Systems (United States) 
9148 4K Benchmarking hardware architecture candidates for the NFIRAOS real-time controller [9148-176]

M. Smith, D. Kerley, G. Herriot, J.-P. Véran, NRC - Dominion Astrophysical Observatory (Canada)

$91484 \mathrm{~L}$ Kalman filter design for atmospheric tip/tilt, tip/tilt anisoplanatism and focus filtering on extremely large telescopes [9148-177]

L. Gilles, Thirty Meter Telescope Observatory Corp. (United States); H. F. Raynaud, Lab. Charles Fabry de I'Institut d'Optique, CNRS, Univ. Paris-Sud (France); C. Correia, Univ. do Porto (Portugal); L. Wang, B. Ellerbroek, C. Boyer, Thirty Meter Telescope Observatory Corp. (United States); C. Kulcsár, Lab. Charles Fabry de I'Institut d'Optique, CNRS, Univ. Paris-Sud (France)

$91484 \mathrm{M}$ First on-sky results of a neural network based tomographic reconstructor: Carmen on Canary [9148-178]

J. Osborn, Durham Univ. (United Kingdom); D. Guzman, Pontificia Univ. Católica de Chile (Chile); F. J. de Cos Juez, Univ. de Oviedo (Spain); A. G. Basden, T. J. Morris, Durham Univ. (United Kingdom); É. Gendron, LESIA, CNRS, Observatoire de Paris (France); T. Butterley, R. M. Myers, Durham Univ. (United Kingdom); A. Guesalaga, Pontificia Univ. Católica de Chile (Chile); F. Sanchez Lasheras, M. Gomez Victoria, M. L. Sánchez Rodríguez, Univ. de Oviedo (Spain); D. Gratadour, G. Rousset, LESIA, CNRS, Observatoire de Paris (France)

$91484 \mathrm{~N} \quad$ Robustness of tomographic reconstructors versus real atmospheric profiles in the ELT perspective [9148-179]

E. Gendron, C. Morel, LESIA, CNRS, Observatorie de Paris (France); J. Osborn, Durham Univ. (United Kingdom); O. Martin, D. Gratadour, F. Vidal, LESIA, CNRS, Observatorie de Paris (France); M. Le Louarn, European Southern Observatory (Germany); G. Rousset, LESIA, CNRS, Observatorie de Paris (France)

POSTER SESSION: POST-PROCESSING AO DATA

914840 Morphology of distant galaxies with MCAO [9148-180]

B. Neichel, Lab. d'Astrophysique de Marseille, CNRS, Aix Marseille Univ. (France);

M. Huertas-Company, T. Huellou, GEPI, CNRS, Observatoire de Paris à Meudon (France); B. Epinat, Lab. d'Astrophysique de Marseille, CNRS, Aix Marseille Univ. (France); M. Puech, GEPI, CNRS, Observatoire de Paris à Meudon (France); D. Gratadour, LESIA, CNRS, Observatoire de Paris (France)

9148 4P Maximum likelihood approach for the adaptive optics point spread function reconstruction [9148-181]

J. Exposito, D. Gratadour, G. Rousset, Y. Clénet, LESIA, CNRS, Observatoire de Paris, Univ. Paris-Diderot (France); L. Mugnier, ONERA (France); É. Gendron, LESIA, CNRS, Observatoire de Paris, Univ. Paris-Diderot (France) 
$91484 Q \quad$ CHARA array adaptive optics II: non-common-path correction and downstream optics [9148-183]

T. ten Brummelaar, The CHARA Array (United States); X. Che, Univ. of Michigan

(United States); H. McAlister, CHARA, Georgia State Univ. (United States); M. Ireland, The

Australian National Univ. (Australia); J. Monnier, Univ. of Michigan (United States);

D. Mourard, Univ. de Nice (France); S. Ridgway, National Optical Astronomy Observatory

(United States); J. Sturmann, L. Sturmann, N. Turner, The CHARA Array (United States);

P. Tuthill, The Univ. of Sydney (Australia)

9148 4S Laser guide star adaptive optics point spread function reconstruction project at W. M. Keck Observatory: preliminary on-sky results [9148-185]

L. Jolissaint, Univ. of Applied Sciences Western Switzerland (Switzerland); S. Ragland,

P. Wizinowich, W. M. Keck Observatory (United States); A. Bouxin, Univ. of Applied Sciences Western Switzerland (Switzerland)

9148 4T Real-time Strehl and image quality performance estimator at Paranal Observatory [9148-186]

D. Mawet, A. Smette, European Southern Observatory (Chile); M. S. Sarazin, H. Kuntschner, European Southern Observatory (Germany); J. H. Girard, European Southern Observatory (Chile)

$91484 \mathrm{U}$ Strehl-constrained reconstruction of post-adaptive optics data and the Software Package AIRY, v. 6.1 [9148-187]

M. Carbillet, Observatoire de la Cote d'Azur, CNRS, Univ. de Nice Sophia-Antipolis (France); A. La Camera, Univ. degli Studi di Genova (Italy); J. Deguignet, Observatoire de la Cote d'Azur, CNRS, Univ. de Nice Sophia-Antipolis (France); M. Prato, Univ. degli Studi di Modena e Reggio Emilia (Italy); M. Bertero, Univ. degli Studi di Genova (Italy); É. Aristidi, Observatoire de la Cote d'Azur, CNRS, Univ. de Nice Sophia-Antipolis (France); P. Boccacci, Univ. degli Studi di Genova (Italy)

9148 4V On-sky PSF reconstruction with APETy [9148-188]

R. Olguin, Pontificia Univ. Católica de Chile (Chile); M. Hartung, T. Hayward, Gemini Observatory (Chile); D. Gratadour, LESIA, CNRS, Observatorie de Paris (France);

A. Guesalaga, Pontificia Univ. Católica de Chile (Chile)

9148 4W Analysis of turbulent atmospheric anisoplanatism influence on adaptive optics system over horizontal path [9148-253]

Q. Sun, Y. Ning, B. Shu, S. Du, National Univ. of Defense Technology (China)

\section{POSTER SESSION: NEW PROPOSED AO SYSTEMS}

$91484 \mathrm{X}$ Meaningful options for a dichroic unit within the natural \& laser guide star AO systems at the Giant Magellan Telescope [9148-189]

J. Antichi, E. Pinna, S. Esposito, M. Bonaglia, L. Busoni, INAF - Osservatorio Astrofisico di Arcetri (Italy); F. Santoro, A. Bouchez, Giant Magellan Telescope Corp. (United States)

$91484 Z$ Design of adaptive optics calibration source for the Giant Magellan Telescope [9148-192]

P. Zhou, J. H. Burge, C. Zhao, S. Benjamin, B. Cuerden, College of Optical Sciences, The Univ. of Arizona (United States); A. Bouchez, Giant Magellan Telescope Corp.

(United States) 
914850 Optical design of the Big Bear Solar Observatory's multi-conjugate adaptive optics system [9148-193]

X. Zhang, N. Gorceix, Big Bear Solar Observatory (United States); D. Schmidt, National Solar Observatory (United States); P. R. Goode, W. Cao, Big Bear Solar Observatory (United States); T. R. Rimmele, National Solar Observatory (United States); R. Coulter, Big Bear Solar Observatory (United States)

$914851 \quad 1500 \mathrm{~Hz}$ adaptive optics system using commercially available components [9148-194] A. Schimpf, M. Micallef, J. Charton, ALPAO S.A.S. (France)

914853 Testing the analytical model of the pyramid wavefront sensor with high-order aberrations on the optical bench [9148-196]

M. Rosensteiner, J.-P. Véran, NRC - Herzburg Institute of Astrophysics (Canada)

\section{POSTER SESSION: WAVEFRONT SENSING}

914855 A composite tracking sensor with high accuracy and large dynamic range [9148-199] X. Ma, K. Wei, W. Zheng, C. Rao, Institute of Optics and Electronics (China) and The Key Lab. Of Adaptive Optics (China)

914856 Theoretical analyses for the relationship between the performance of quadrant photodetector and the size of incident light spot [9148-201]

Z. Zheng, Nanjing Institute of Astronomical Optics \& Technology (China) and Univ. of Chinese Academy of Sciences (China); C. Li, S. Zhang, Nanjing Institute of Astronomical Optics \& Technology (China)

914857 Non-common path aberration corrections for current and future AO systems [91 18-202] M. Lamb, Univ. of Victoria (Canada) and NRC - Herzberg Institute of Astrophysics (Canada); D. R. Andersen, J.-P. Véran, NRC - Herzberg Institute of Astrophysics (Canada); C. Correia, Univ. do Porto (Portugal); G. Herriot, M. Rosensteiner, NRC - Herzberg Institute of Astrophysics (Canada); J. Fiege, Univ. of Manitoba (Canada)

914858 Cross-scale inference and wavefront reconstruction [9148-203]

S. K. Maji, INRIA (France); T. Fusco, ONERA (France); H. Yahia, INRIA (France)

914859 Wavefront sensing from the image domain with the Oxford-SWIFT integral field spectrograph [9148-204]

B. Pope, N. Thatte, Univ. of Oxford (United Kingdom); R. Burruss, Jet Propulsion Lab. (United States); M. Tecza, F. Clarke, G. Cotter, Univ. of Oxford (United Kingdom)

9148 5B Near-infrared aberration tracking using a correlation algorithm on the Galactic Center [9148-207]

N. Anugu, P. Garcia, Univ. do Porto (Portugal); A. Amorim, P. Gordo, Univ. de Lisboa (Portugal); F. Eisenhaver, Max-Planck-Institut für extraterrestrische Physik (Germany); G. Perrin, Observatoire de Paris à Meudon, CNRS (France); W. Brandner, Max-Planck-Institut für Astronomie (Germany); C. Straubmeier, Univ. zu Köln (Germany); K. Perraut, Institut de Planétologie et d'Astrophysique de Grenoble, CNRS (France) 
9148 5C High speed and high precision pyramid wavefront sensor: In labs validation and preparation to on sky demonstration [9148-208]

K. El Hadi, Lab. d'Astrophysique de Marseille, CNRS, Aix Marseille Univ. (France); T. Fusco, J.-F. Sauvage, ONERA (France) and Lab. d'Astrophysique de Marseille, CNRS, Aix Marseille Univ. (France); B. Neichel, Lab. d'Astrophysique de Marseille, CNRS, Aix Marseille Univ. (France)

9148 5D Focal-plane wavefront sensing with high-order adaptive optics systems [9148-209] V. Korkiakoski, Delft Ctr. for Systems and Control (Netherlands) and Leiden Observatory (Netherlands); C. U. Keller, Leiden Observatory (Netherlands); N. Doelman, TNO Science and Industry (Netherlands) and Leiden Observatory (Netherlands); M. Kenworthy, G. Otten, Leiden Observatory (Netherlands); M. Verhaegen, Delft Ctr. for Systems and Control (Netherlands)

$91485 \mathrm{E} \quad$ A novel means of measuring non-common path errors in an adaptive optics system [9148-210]

E. E. Bloemhof, National Science Foundation (United States)

9148 5G Pre-shipment test of the ARGOS laser guide star wavefront sensor [9148-212] M. Bonaglia, L. Busoni, T. Mazzoni, A. Puglisi, J. Antichi, S. Esposito, INAF - Osservatorio Astrofisico di Arcetri (Italy); G. Orban de Xivry, S. Rabien, Max-Planck-Institut für extraterrestrische Physik (Germany)

$91485 \mathrm{H}$ Design optimization and lab demonstration of ZELDA: a Zernike sensor for nearcoronagraph quasi-static measurements [9148-213]

M. N'Diaye, Space Telescope Science Institute (United States); K. Dohlen, A. Caillat, A. Costille, Lab. d'Astrophysique de Marseille, CNRS, Aix-Marseille Univ. (France); T. Fusco, Lab. d'Astrophysique de Marseille, CNRS, Aix-Marseille Univ. (France) and ONERA (France); A. Jolivet, Lab. d'Astrophysique de Marseille, CNRS, Aix-Marseille Univ. (France) and Institut d'Astrophysique et de Géophysique (Belgium); F. Madec, Lab. d'Astrophysique de Marseille, CNRS, Aix-Marseille Univ. (France); L. Mugnier, ONERA (France); B. Paul, J.-F. Sauvage, Lab. d'Astrophysique de Marseille, CNRS, Aix-Marseille Univ. (France) and ONERA (France); R. Soummer, Space Telescope Science Institute (United States); A. Vigan, Lab. d'Astrophysique de Marseille, CNRS, Aix-Marseille Univ. (France); J. K. Wallace, Jet Propulsion Lab. (United States)

$914851 \quad$ Understanding and correcting low order residual static aberrations in adaptive optics corrected images [9148-214]

R. Rampy, S. Ragland, P. Wizinowich, R. Campbell, W. M. Keck Observatory (United States)

9148 5J Applications of variable focus liquid lenses for curvature wave-front sensors in astronomy [9148-215]

J. Fuentes-Fernández, S. Cuevas, L. C. Alvarez-Nuñez, A. M. Watson, Univ. Nacional

Autónoma de México (Mexico)

$91485 \mathrm{~K}$ Comparing the performance of open loop centroiding techniques in the Raven MOAO system [9148-216]

D. R. Andersen, NRC - Herzberg Institute of Astrophysics (Canada); C. Bradley, D. Gamroth, Univ. of Victoria (Canada); D. Kerley, NRC - Herzberg Institute of Astrophysics (Canada); O. Lardière, Univ. of Victoria (Canada); J.-P. Véran, NRC - Herzberg Institute of Astrophysics (Canada) 
$91485 \mathrm{~L} \quad$ Effects of differential wavefront sensor bias drifts on high contrast imaging [9148-217] N. Sadakuni, Gemini Observatory (United States); B. A. Macintosh, Kavli Institute for Particle Astrophysics and Cosmology, Stanford Univ. (United States) and Lawrence Livermore National Lab. (United States); D. W. Palmer, L. A. Poyneer, Lawrence Livermore National Lab. (United States); C. E. Max, Univ. of California, Santa Cruz (United States); D. Savransky, Cornell Univ. (United States); S. J. Thomas, NASA Ames Research Ctr. (United States); A. Cardwell, S. Goodsell, M. Hartung, P. Hibon, F. Rantakyrö, A. Serio, Gemini Observatory (Chile)

$91485 \mathrm{M}$ Wavefront sensing in a partially illuminated, rotating pupil [9148-218]

T. Bertram, K. Kumar Radhakrishnan Santhakumari, Max-Planck-Institut für Astronomie (Germany); L. Marafatto, INAF - Osservatorio Astronomico di Padova (Italy) and Univ. delgi Studi di Padova (Italy); C. Arcidiacono, INAF - Osservatorio Astronomico di Bologna (Italy); J. Berwein, Max-Planck-Institut für Astronomie (Germany); R. Ragazzoni, INAF - Osservatorio Astronomico di Padova (Italy); T. M. Herbst, Max-Planck-Institut für Astronomie (Germany)

$91485 \mathrm{~N} \quad$ A new phase retrieval algorithm based on multi-layered intensity distribution [9148-220] S. Du, X. Zhang, B. Shu, National Univ. of Defense Technology (China)

$914850 \quad$ New CCD imagers for adaptive optics wavefront sensors [9148-221]

D. R. Schuette, R. K. Reich, I. Prigozhin, B. E. Burke, MIT Lincoln Lab. (United States); R. Johnson, Air Force Research Lab. (United States)

$91485 \mathrm{P} \quad$ Discretized aperture mapping with a micro-lenses array for interferometric direct imaging [9148-222]

F. Patru, J. Antichi, Osservatorio Astrofisico di Arcetri (Italy); D. Mawet, European Southern Observatory (Chile); L. Jolissaint, Haute Ecole d'Ingenierie et de Gestion du Canton de Vaud (Switzerland); M. Carbillet, Observatoire de la Côte d'Azur, CNRS (France); J. Milli, J. Girard, European Southern Observatory (Chile); P. Rabou, Institut de Planetologie et d'Astrophysique, CNRS (France); E. Giro, Osservatorio Astronomico di Padova (Italy);

D. Mourard, Observatoire de la Cote d'Azur, CNRS (France)

9148 5Q On-sky low order non-common path correction of the GPI calibration unit [9148-224] M. Hartung, Gemini Observatory (Chile); B. Macintosh, Kavli Institute for Particle Astrophysics and Cosmology, Stanford Univ. (United States); P. Langlois, N. Sadakuni, Gemini Observatory (Chile); D. Gavel, Lick Observatory, Univ. of California, Santa Cruz (United States); J. K. Wallace, Jet Propulsion Lab. (United States); D. Palmer, L. Poyneer, Lawrence Livermore National Lab. (United States); D. Savransky, Cornell Univ. (United States); S. Thomas, NASA Ames Research Ctr. (United States); D. Dillon, Lick Observatory, Univ. of California, Santa Cruz (United States); J. Dunn, NRC - Herzburg Institute of Astrophysics (Canada); P. Hibon, F. Rantakyrö, S. Goodsell, Gemini Observatory (Chile)

POSTER SESSION: PATHFINDERS TO ENABLE AO ON ELTS AND NEW AO CONCEPTS

9148 5S HALOS: fast, autonomous, holographic adaptive optics [9148-226] G. P. Andersen, P. Gelsinger-Austin, R. Gaddipati, P. Gaddipati, F. Ghebremichael, U.S. Air Force Academy (United States)

$91485 \mathrm{~T} \quad$ A laser tomography test bed for extremely large telescopes [9148-227] R. Conan, P. Piatrou, F. Rigaut, The Australian National Univ. (Australia); K. Uhlendorf, The Australian National Univ. (Germany) 
$91485 \mathrm{U}$ DRAGON, the Durham real-time, tomographic adaptive optics test bench: progress and results [9148-228]

A. P. Reeves, R. M. Myers, T. J. Morris, A. G. Basden, N. A. Bharmal, S. Rolt, D. G. Bramall, N. A. Dipper, E. J. Younger, Durham Univ. (United Kingdom)

$91485 \mathrm{~V}$ CHOUGH, the Canary Hosted-Upgrade for High-Order Adaptive Optics [9148-229] N. A. Bharmal, R. M. Myers, A. G. Basden, D. Hölck, T. J. Morris, Durham Univ. (United Kingdom)

9148 5W CRAO: a compact and refractive adaptive-optics [9148-230]

N. Fujishiro, E. Kitao, Kyoto Sangyo Univ. (Japan); T. Shimizu, Nishimura Co., Ltd. (Japan); T. Matsui, Kyoto Sangyo Univ. (Japan); Y. Ikeda, Kyoto Sangyo Univ. (Japan) and Photocoding (Japan); H. Kawakita, Kyoto Sangyo Univ. (Japan); S. Oya, Subaru Telescope, National Astronomical Observatory of Japan (United States)

9148 5X Pyramidal Wavefront Sensor Demonstrator at INO [9148-231]

O. Martin, Institut National d'Optique (Canada); J.-P. Véran, NRC - Herzberg Institute of Astrophysics (Canada); G. Anctil, P. Bourqui, F. Châteauneuf, J. Gauvin, P. Goyette, F. Lagacé, S. Turbide, M. Wang, Institut National d'Optique (Canada)

$91485 Y \quad$ An MCAO test bench for NFIRAOS [9148-232]

P. Turri, Univ. of Victoria (Canada) and NRC - Herzburg Institute of Astrophysics (Canada): D. R. Andersen, J.-P. Véran, P. Spanò, M. Rosensteiner, E. A. McVeigh, NRC - Herzburg Institute of Astrophysics (Canada)

\section{POSTER SESSION: CHARACTERIZATION, MEASUREMENT AND MODELING OF THE} DISTURBANCES FACED BY AO

914860 Isoplanatic patch considerations for solar telescope multi-conjugate adaptive optics [9148-233]

J. M. Beckers, College of Optical Sciences, The Univ. of Arizona (United States) and Kiepenhever-Institute für Sonnenphysik (Germany)

914862 Simulation of the imaging quality of ground-based telescopes affected by atmospheric disturbances [9148-235]

Y. Ren, Nanjing Institute of Astronomical Optics \& Technology (China) and Univ. Of Chinese Academy of Sciences (China); S. Kou, B. Gu, Nanjing Institute of Astronomical Optics \& Technology (China)

914863 Towards a reliability assessment of the $\mathrm{C}^{2} \mathrm{~N}$ and wind speed vertical profiles retrieved from GeMS [9148-236]

B. Neichel, Lab. d'Astrophysique de Marseille, CNRS, Aix Marseille Univ. (France); E. Masciadri, INAF - Osservatorio Astrofisico di Arcetri (Italy); A. R. Guesalaga, Pontificia Univ. Católica de Chile (Chile); F. Lascaux, INAF - Osservatorio Astrofisico di Arcetri (Italy); C. Béchet, Pontificia Univ. Católica de Chile (Chile)

914864 Validation tests of the AOF $\mathrm{C}_{\mathrm{n}}{ }^{2}$ profiler [9148-237]

A. Garcia Rissmann, European Southern Observatory (Germany); A. Guesalaga, Pontificia Univ. Católica de Chile (Chile); J. Kolb, M. Le Louarn, P.-Y. Madec, R. Arsenault, European Southern Observatory (Germany) 
914865 MOSE: verification of Meso-NH forecasts of the atmospheric surface parameters at Cerro Paranal and Cerro Armazones using contingency tables [9148-238]

F. Lascaux, E. Masciadri, L. Fini, INAF - Osservatorio Astrofisico di Arcetri (Italy)

914866 Statistics of atmospheric turbulence at Cerro Pachon using the GeMS profiler [9148-240] I. Rodriguez, Pontificia Univ. Católica de Chile (Chile); B. Neichel, Gemini Observatory (Chile) and Lab. d'Astrophysique de Marseille, CNRS, Aix Marseille Univ. (France); C. Béchet, D. Guzmán, A. Guesalaga, Pontificia Univ. Católica de Chile (Chile)

914867 Extremely high-resolution ground-layer optical turbulence profile at Mauna Kea [9148-241] M. R. Chun, Institute for Astronomy, Univ. of Hawai'i (United States); O. Lai, Gemini Observatory (United States); T. Butterley, Durham Univ. (United Kingdom); S. Goebel, C. Baranec, Institute for Astronomy, Univ. of Hawai'i (United States); D. Toomey, Mauna Kea Infrared LLC (United States)

914868 Aero-thermal simulations of the TMT Laser Guide Star Facility [9148-243]

K. Vogiatzis, C. Boyer, Thirty Meter Telescope Observatory Corp. (United States); K. Wei, J. Tang, Institute of Optics and Electronics (China); B. Ellerbroek, Thirty Meter Telescope Observatory Corp. (United States)

914869 Usage of Large Binocular Telescope pupils to estimate the local outer scale of turbulence [9148-244]

J. C. Guerra, Large Binocular Telescope Observatory (United States) and Steward Observatory (United States); A. Otarola, Thirty Meter Telescope Observatory Corp. (United States); G. Brusa, Large Binocular Telescope Observatory (United States) and Steward Observatory, The Univ. of Arizona (United States); V. Bailey, Steward Observatory, The Univ. of Arizona (United States); P. Hinz, Large Binocular Telescope Observatory (United States) and Steward Observatory (United States); E. Pinna, INAF - Osservatorio Astrofisico di Arcetri (Italy)

9148 6A Implementation of SLODAR atmospheric turbulence profiling to the ARGOS system [9148-245]

T. Mazzoni, INAF - Osservatorio Astrofisico di Arcetri (Italy) and Univ. degli studi di Firenze (Italy); L. Busoni, M. Bonaglia, S. Esposito, INAF - Osservatorio Astrofisico di Arcetri (Italy)

POSTER SESSION: AO MODELING, ANALYSIS AND SIMULATIONS

9148 6B Integrated modeling of the GMT laser tomography adaptive optics system [9148-247] P. Piatrou, The Australian National Univ. (Australia)

9148 6C Object-oriented Matlab adaptive optics toolbox [9148-248] R. Conan, The Australian National Univ. (Australia); C. Correia, Univ. do Porto (Portugal)

9148 6D Modeling the effect of high altitude turbulence in wide-field correlating wavefront sensing and its impact on the performance of solar AO systems [9148-249] I. Montilla, Instituto de Astrofísica de Canarias (Spain); M. Tallon, M. Langlois, Ctr. de Recherche Astronomique de Lyon (France); C. Béchet, Pontificia Univ. Católica de Chile (Chile); M. Collados Vera, Instituto de Astrofísica de Canarias (Spain) 
$91486 \mathrm{~F}$ End to end numerical simulations of the MAORY multiconjugate adaptive optics system [9148-251]

C. Arcidiacono, L. Schreiber, G. Bregoli, E. Diolaiti, I. Foppiani, INAF - Osservatorio Astronomico di Bologna (Italy); G. Cosentino, Univ. degli Studi di Bologna (Italy);

M. Lombini, INAF - Osservatorio Astronomico di Bologna (Italy); R. C. Butler, INAF - Istituto di Astrofisica Spaziale e Fisica Cosmica (Italy); P. Ciliegi, INAF - Osservatorio Astronomico di Bologna (Italy)

$91486 G \quad$ ULTIMATE-SUBARU: simulation update [9148-252]

S. Oya, Y. Hayano, O. Lai, I. Iwata, Subaru Telescope, National Astronomical Observatory of Japan (United States); T. Kodama, National Astronomical Observatory of Japan (Japan); N. Arimoto, Y. Minowa, Subaru Telescope, National Astronomical Observatory of Japan (United States); M. Akiyama, Y. H. Ono, Tohoku Univ. (Japan); H. Terada, T. Usuda, Subaru Telescope, National Astronomical Observatory of Japan (United States); H. Takami, National Astronomical Observatory of Japan (Japan); T. Nishimura, N. Takato, D. Tomono, Subaru Telescope, National Astronomical Observatory of Japan (United States)

$91486 \mathrm{H} \quad$ Modelling global multi-conjugated adaptive optics [9148-270]

V. Viotto, R. Ragazzoni, D. Magrin, M. Bergomi, M. Dima, J. Farinato, INAF - Osservatorio Astronomico di Padova (Italy); L. Marafatto, D. Greggio, INAF - Osservatorio Astronomico di Padova (Italy) and Univ. degli Studi di Padova (Italy)

$914861 \quad$ Adaptive optics simulations for the MICADO SCAO system [9148-254]

F. Vidal, E. Gendron, Y. Clénet, D. Gratadour, G. Rousset, LESIA, CNRS, Observatorie de Paris, Univ. Paris Diderot (France); R. Davies, Max-Planck-Institut für extraterrestrische Physik (Germany)

9148 6J AO modelling for wide-field E-ELT instrumentation using Monte-Carlo simulation [9148-255] A. Basden, S. Morris, T. Morris, R. Myers, Durham Univ. (United Kingdom)

$91486 \mathrm{~K}$ Optical design trade-offs of the multi conjugate adaptive optics relay for the European Extremely Large Telescope [9148-256]

M. Lombini, E. Diolaiti, INAF - Osservatorio Astronomico di Bologna (Italy); A. De Rosa, INAF Istituto di Astrofisica Spaziale e Fisica cosmica Bologna (Italy)

$91486 \mathrm{~L}$ A novel fast and accurate pseudo-analytical simulation approach for MOAO [9148-257]

É. Gendron, LESIA, Observatoire de Paris, CNRS, Univ. Paris Diderot (France); A. Charara,

A. Abdelfattah, King Abdullah Univ. of Science and Technology (Saudi Arabia);

D. Gratadour, LESIA, Observatoire de Paris, CNRS, Univ. Paris Diderot (France); D. Keyes,

H. Ltaief, King Abdullah Univ. of Science and Technology (Saudi Arabia); C. Morel, F. Vidal, A. Sevin, G. Rousset, LESIA, Observatoire de Paris, CNRS, Univ. Paris Diderot (France)

$91486 \mathrm{M}$ TMT-AGE: numerical simulation of a new tomographic reconstruction method for wide FoR MOAO [9148-258]

Y. H. Ono, Tohoku Univ. (Japan) and Subaru Telescope, National Astronomical Observatory of Japan (United States); M. Akiyama, Tohoku Univ. (Japan); S. Oya, Subaru Telescope,

National Astronomical Observatory of Japan (United States)

$91486 \mathrm{~N}$ A numerical exploration of phase-retrieval error-metric surfaces [9148-259]

A. S. Jurling, J. R. Fienup, The Institute of Optics, Univ. of Rochester (United States) 
914860 COMPASS: an efficient, scalable and versatile numerical platform for the development of ELT AO systems [9148-260]

D. Gratadour, LESIA, Observatoire de Paris, CNRS, Univ. Paris Diderot (France); M. Puech, GEPI, Observatoire de Paris, CNRS, Univ. Paris Diderot (France); C. Vérinaud, IPAG, CNRS, Univ. Joseph Fourier (France); P. Kestener, Maison de la Simulation, CNRS, Univ. Paris Sud, Univ. de Versailles (France); M. Gray, Lab. d'Astrophysique de Marseille, CNRS, Univ. d'AixMarseille (France); C. Petit, ONERA (France); J. Brulé, Y. Clénet, F. Ferreira, E. Gendron, M. Lainé, A. Sevin, G. Rousset, LESIA, Observatoire de Paris, CNRS, Univ. Paris Diderot (France); F. Hammer, I. Jégouzo, M. Paillous, S. Taburet, Y. Yang, GEPI, Observatoire de Paris, CNRS, Univ. Paris Diderot (France); J.-L. Beuzit, A. Carlotti, M. Westphal, IPAG, CNRS, Univ. Joseph Fourier (France); B. Epinat, M. Ferrari, T. Gautrais, J. C. Lambert, B. Neichel, S. Rodionov, Lab. d'Astrophysique de Marseille, CNRS, Univ. d'Aix-Marseille (France)

$91486 \mathrm{P}$ The effects of AO systems on polarized light [9148-261]

M. Stangalini, INAF - Osservatorio Astronomico di Roma (Italy); L. Giovannelli, D. Del Moro, F. Berrilli, R. Piazzesi, Univ. degli Studi di Roma Tor Vergata (Italy)

$91486 Q \quad$ Impact of sodium layer variations on the performance of the E-ELT MCAO module [9148-262]

L. Schreiber, E. Diolaiti, C. Arcidiacono, INAF - Osservatorio Astronomico di Bologna (Italy); T. Pfrommer, R. Holzlöhner, European Southern Observatory (Germany); M. Lombini, INAF Osservatorio Astronomico di Bologna (Italy); P. Hickson, The Univ. of British Columbia (Canada)

$91486 R \quad$ Modelization of a pyramid wavefront sensor for the E-ELT in the context of the COMPASS project [9148-263]

A. Carlotti, C. Vérinaud, IPAG, CNRS, Univ. Joseph Fourier (France); D. Gratadour, LESIA, CNRS, Observatoire de Paris (France); M. Westphal, J.-L. Beuzit, IPAG, CNRS, Univ. Joseph Fourier (France)

9148 6T Modeling instrumental field-dependent aberrations in the NIRC2 instrument on the Keck II telescope [9148-265]

B. N. Sitarski, G. Witzel, M. P. Fitzgerald, L. Meyer, A. M. Ghez, Univ. of California, Los Angeles (United States); R. D. Campbell, W. M. Keck Observatory (United States); J. R. Lu, Institute for Astronomy, Univ. of Hawai'i (United States); K. Matthews, California Institute of Technology (United States); P. Wizinowich, J. Lyke, W. M. Keck Observatory (United States)

$91486 \mathrm{~V} \quad$ Quasi-realtime solar adaptive optics simulation [9148-267]

J. Marino, D. Schmidt, National Solar Observatory (United States)

9148 6W Remembrance of phases past: An autoregressive method for generating realistic atmospheres in simulations [9148-268]

S. Srinath, Univ. of California, Santa Cruz (United States); L. A. Poyneer, Lawrence Livermore National Lab. (United States); A. R. Rudy, Univ. of California, Santa Cruz (United States); S. M. Ammons, Lawrence Livermore National Lab. (United States)

Author Index

xxxiv 


\title{
Conference Committee
}

\author{
Symposium Chairs
}

Luc Simard, National Research Council Canada (Canada)

Gillian S. Wright, UK Astronomy Technology Centre (United Kingdom)

Symposium Co-chairs

Colin Cunningham, UK Astronomy Technology Centre (United Kingdom)

Masanori lye, National Astronomical Observatory of Japan (Japan)

\section{Conference Chairs}

Enrico Marchetti, European Southern Observatory (Germany) Laird M. Close, The University of Arizona (United States)

Jean-Pierre Véran, NRC - Herzberg (Canada)

\section{Conference Program Committee}

Emiliano Diolaiti, INAF - Osservatorio Astronomico di Bologna (Italy) Celine D'Orgeville, Research School of Astronomy \& Astrophysics, Australian National University (Australia)

Brent L. Ellerbroek, Thirty Meter Telescope Observatory Corporation (United States)

Simone Esposito, INAF - Osservatorio Astrofisico di Arcetri (Italy) Thierry Fusco, ONERA (France)

Donald Gavel, University of California, Santa Cruz (United States)

Michael Hart, Hart Scientific Consulting International L.L.C. (United States)

Yutaka Hayano, Subaru Telescope, National Astronomical Observatory of Japan (United States)

Glen Herriot, NRC - Herzberg (Canada)

Norbert Hubin, European Southern Observatory (Germany)

Markus Kasper, European Southern Observatory (Germany)

Caroline Kulcsar, Institut d'Optique (France)

Anne-Marie Lagrange, Institut de Planétologie et d'Astrophysique de Grenoble (France)

Bruce A. Macintosh, Lawrence Livermore National Laboratory (United States)

Pierre-Yves Madec, European Southern Observatory (Germany)

Richard M. Myers, Durham University (United Kingdom)

Francois Rigaut, The Australian National University (Australia) 
Andrei Tokovinin, National Optical Astronomy Observatory (United States)

Mitchell Troy, Jet Propulsion Laboratory (United States)

Peter L. Wizinowich, W. M. Keck Observatory (United States)

\section{Session Chairs}

Monday Plenary Session

Luc Simard, National Research Council Canada (Canada)

Tuesday Plenary Session

Gillian S. Wright, UK Astronomy Technology Centre (United Kingdom)

Wednesday Plenary Session

Colin Cunningham, UK Astronomy Technology Centre (United Kingdom)

Thursday Plenary Session

Masanori lye, National Astronomical Observatory of Japan (Japan)

1 Status of Current AO Instrument Projects I

Enrico Marchetti, European Southern Observatory (Germany)

2 Laser Guide Star Systems I

Celine D'Orgeville, Research School of Astronomy \& Astrophysics (Australia)

3 Astronomy with $\mathrm{AO}$ I

Laird M. Close, The University of Arizona (United States)

4 Wavefront Correction I

Norbert Hubin, European Southern Observatory (Germany)

5 Extreme $\mathrm{AO}$ I

Simone Esposito, INAF - Osservatorio Astrofisico di Arcetri (Italy)

6 Advances in AO Control I

Caroline Kulcsar, Institut d'Optique Graduate School (France)

7 Post-Processing AO Data I

Francois Rigaut, Research School of Astronomy \& Astrophysics (Australia)

8 AO for ELTS

Richard M. Myers, Durham University (United Kingdom) 
9 New Proposed AO Systems and Concepts for Large Telescopes and ELTS

Simone Esposito, INAF - Osservatorio Astrofisico di Arcetri (Italy)

10 Wavefront Sensing I

Jean-Pierre Véran, NRC - Herzberg (Canada)

11 Laser Guide Star Systems II

Peter L. Wizinowich, W. M. Keck Observatory (United States)

12 Pathfinders to Enable AO on ELTs and New AO Concepts I

Glen Herriot, NRC - Herzburg (Canada)

13 Pathfinders to Enable AO on ELTs and New AO Concepts II

Glen Herriot, NRC - Herzburg (Canada)

14 Astronomy with $\mathrm{AO}$ II

Anne-Marie Lagrange, Institut de Planétologie et d'Astrophysique de Grenoble (France)

15 Status of Current AO Instrument Projects II

Emiliano Diolaiti, INAF - Osservatorio Astronomico di Bologna (Italy)

16 Characterization, Measurement and Modeling of the Distrurbances Faced by AO

Michael Hart, Hart Scientific Consulting International L.L.C. (United States)

17 Extreme $\mathrm{AO} \|$

Bruce A. Macintosh, Lawrence Livermore National Laboratory (United States)

18 Wavefront Correction II

Norbert Hubin, European Southern Observatory (Germany)

19 Status of Current AO Instrument Projects III

Yutaka Hayano, Subaru Telescope, National Astronomical Observatory of Japan (United States)

20 Wavefront Sensing II

Donald Gavel, University of California, Santa Cruz (United States)

21 Advances in AO Control II

Mitchell Troy, Jet Propulsion Laboratory (United States)

22 AO Modeling, Analysis and Simulations

Thierry Fusco, ONERA (France) 
23 Post-Processing AO Data II

Jean-Pierre Véran, NRC - Herzburg (Canada)

xxxviii

Proc. of SPIE Vol. 9148 914801-38

Downloaded From: https://www.spiedigitallibrary.org/conference-proceedings-of-spie on 25 Apr 2023 Terms of Use: https://www.spiedigitallibrary.org/terms-of-use 